\title{
ON CHROMATIC UNIQUENESS OF SOME COMPLETE TRIPARTITE GRAPHS
}

\author{
Pavel A. Gein \\ Ural Federal University, \\ 51, Lenin ave., Ekaterinburg, 620000, Russia \\ pavel.gein@gmail.com
}

\begin{abstract}
Let $P(G, x)$ be a chromatic polynomial of a graph $G$. Two graphs $G$ and $H$ are called chromatically equivalent iff $P(G, x)=H(G, x)$. A graph $G$ is called chromatically unique if $G \simeq H$ for every $H$ chromatically equivalent to $G$. In this paper, the chromatic uniqueness of complete tripartite graphs $K\left(n_{1}, n_{2}, n_{3}\right)$ is proved for $n_{1} \geqslant n_{2} \geqslant n_{3} \geqslant 2$ and $n_{1}-n_{3} \leqslant 5$.
\end{abstract}

Keywords: Chromatic uniqueness, Chromatic equivalence, Complete multipartite graphs, Chromatic polynomial.

\section{Introduction}

All graphs in this paper are finite and simple, i.e., they do not contain loops and multiple edges. Basic terminology is used according to [1].

Let $G=(V, E)$ be a graph with a vertex set $V$ and an edge set $E$. A coloring of the graph $G$ with $x$ colors is a map $\varphi: V \rightarrow\{1,2, \ldots, x\}$ such that $\varphi(u) \neq \varphi(v)$ for any two adjacent vertices $u$ and $v$ of the graph $G$. We will call the numbers $1,2, \ldots, x$ the colors. A graph is called $x$-colorable if there exists its coloring with $x$ colors. The smallest integer $x$ for which $G$ is $x$-colorable is called the chromatic number of $G$ and is denoted by $\chi(G)$. The number of colorings of a graph $G$ with $x$ colors is denoted by $P(G, x)$. It is well known (see, for example, [1]) that the function $P(G, x)$ is a polynomial in variable $x$. Two graphs $G$ and $H$ are called chromatically equivalent if $P(G, x)=P(H, x)$. A graph $G$ is called chromatically unique if, for any graph $H$, the graphs $G$ and $H$ are chromatically equivalent iff they are isomorphic. Much attention of researches was drawn to the problem of chromatic uniqueness of complete multipartite graphs $K\left(n_{1}, n_{2}, \ldots, n_{t}\right)$. Here are some results, a more complete list can be found in the survey [16] and the monograph [6].

(1) A graph $K\left(n_{1}, n_{2}\right)$, where $n_{1} \geqslant n_{2} \geqslant 2$, is chromatically unique (see [11]).

(2) A graph $K\left(n_{1}, n_{2}, \ldots, n_{t-1}, 1\right)$ is chromatically unique iff $n_{i} \leqslant 2$ for all $i=1,2, \ldots t-1$ (see [14]).

(3) A graph $K\left(n_{1}, n_{2}, \ldots, n_{t}\right)$, where $n_{1} \geqslant n_{2} \geqslant \ldots \geqslant n_{t} \geqslant 2$, is chromatically unique if $n_{1}-n_{t} \leqslant 4$ (see $[2,4,12,13,15]$ ).

The main result of this paper is the following theorem.

Theorem 1. A complete tripartite graph $K\left(n_{1}, n_{2}, n_{3}\right)$ is chromatically unique if $n_{1} \geqslant n_{2} \geqslant$ $n_{3} \geqslant 2$ and $n_{1}-n_{3} \leqslant 5$.

The chromatic uniqueness of a graph $K\left(n_{1}, n_{2}, n_{3}\right)$ with $n_{1} \geqslant n_{2} \geqslant n_{3} \geqslant 2$ and $n_{1}-n_{3} \leqslant 4$ was proved in $[2,12,13]$. The chromatic uniqueness of a graph $K\left(n_{1}, n_{2}, n_{3}\right)$ with $n_{1} \geqslant n_{2} \geqslant n_{3} \geqslant 2$, 
$n_{1}-n_{3}=5$, and $n_{1}+n_{2}+n_{3} \not \equiv 2(\bmod 3)$ was proved in [9]. The main aim of this paper is to prove the theorem in the case $n_{1}-n_{3}=5$ and $n_{1}+n_{2}+n_{3} \equiv 2(\bmod 3)$.

One of the most important tools for studying chromatic equivalence and chromatic uniqueness is chromatic invariants. Assume that a number is assigned to every graph. This number is called a chromatic invariant if it is the same for all chromatically equivalent graphs. For a chromatic invariant $\alpha(G)$ and two arbitrary graphs $G_{1}$ and $G_{2}$, let $\Delta \alpha\left(G_{2}, G_{1}\right)=\alpha\left(G_{2}\right)-\alpha\left(G_{1}\right)$. It is well known (see, for example, [1] or [7]) that the number of vertices, the number of edges, the number of connected components, and the number of triangles are chromatic invariants.

According to Zykov's theorem (see, for example, [1]), the chromatic polynomial can be written as

$$
P(G, x)=\sum_{i=\chi}^{n} p t(G, i) x^{(i)},
$$

where $p t(G, i)$ is the number of partitions of the vertex set of the graph $G$ into $t$ independent sets, and $x^{(i)}$ is the falling factorial of $x$, i.e., $x^{(i)}=x(x-1) \ldots(x-i+1)$. It follows from Zykov's theorem that the numbers $p t(G, i), i=\chi, \ldots, n$, are chromatic invariants. We are most interested in $p t(G, \chi+1)$, which we will write simply as $p t(G)$.

The rest of the paper is structured as follows. Section 2 describes a connection between integer partitions and chromatic uniqueness of complete multipartite graphs and presents a schema of the proof. Also, some properties of chromatic invariants are discussed in this section. In Sections 3, 4, and 5, upper bounds of the invariant $p t$ are proved. Section 6 contains the proof of the main theorem.

\section{Integer partition lattice and chromatic invariants}

Let $n$ be a positive integer. A partition of $n$ is a sequence of nonnegative integers $u=\left(u_{1}, u_{2}, \ldots,\right)$ such that $u_{1} \geqslant u_{2} \geqslant \ldots$ and $n=\sum_{i=1}^{\infty} u_{i}$. The length of a partition $u$ is a number $l$ such that $u_{l}>0$ and $u_{l+1}=u_{l+2}=\ldots=0$. Writing a partition, we will often omit its zero elements.

Let $u=\left(u_{1}, u_{2}, \ldots\right)$ and $v=\left(v_{1}, v_{2}, \ldots\right)$ be two partitions of a number $n$. Define $v \unlhd u$ if

$$
\begin{aligned}
v_{1} & \leq u_{1}, \\
v_{1}+v_{2} & \leq u_{1}+u_{2}, \\
\ldots & \\
v_{1}+v_{2}+\ldots+v_{t-1} & \leq u_{1}+u_{2}+\ldots+u_{t-1},
\end{aligned}
$$

where $t$ is the largest of the lengths of $u$ and $v$. The relation $\unlhd$ is called the dominance order. It was shown in [5] that all partitions of a number $n$ form a lattice with respect to $\unlhd$.

As was proved in [3], all partitions of a number $n$ with fixed length form a lattice with respect to $\unlhd$. Also, Baranskii and Sen'chonok introduced [3] a notion of elementary transformation. A partition $v=\left(v_{1}, v_{2}, \ldots v_{t}\right)$ is a result of an elementary transformation of a partition $u=\left(u_{1}, u_{2}, \ldots, u_{t}\right)$ if there exist indices $i$ and $j$ such that

(1) $1 \leq i<j \leq t$;

(2) $u_{i}-1 \geq u_{i+1}$ and $u_{j-1} \geq u_{j}+1$;

(3) $u_{i}-u_{j}=\delta \geq 2$;

(4) $v_{i}=u_{i}-1, \quad v_{j}=u_{j}+1, \quad u_{k}=v_{k} \quad$ for all $k=1,2, \ldots, t, \quad k \neq i, j$.

It was proved in [3] that $v \unlhd u$ holds if and only if the partition $v$ can be obtained from the partition $u$ by a with finite number of elementary transformations. 
Every complete $t$-partite graph with $n$ vertices can be identified with a partition of length $t$ of the number $n$. Let $u=\left(u_{1}, u_{2}, \ldots, u_{t}\right)$ be a partition of length $t$ of the number $n$. We will write $K(u)$ instead of $K\left(u_{1}, u_{2}, \ldots, u_{t}\right)$ and denote parts of the graph $K(u)$ by $V_{i}$, where $\left|V_{i}\right|=u_{i}$ for all $i=1,2, \ldots, t$.

Let $u$ be a partition of length $t$ of a number $n$. We present the following schema for proving the chromatic uniqueness of the graph $K(u)$. By contradiction, we assume that the graph $K(u)$ is not chromatically unique. This means that there exists a graph $H$ nonisomorphic to the graph $K(u)$, and the graphs $H$ and $K(u)$ are chromatically equivalent. It is clear that the chromatic number of the graph $H$ is $t$; thus, the graph $H$ can be obtained from a complete $t$-partite graph $K(v)$ by removing a set of edges $E$. It was shown in [17] that different complete multipartite graphs are not chromatically equivalent; hence, $E$ must be non-empty. Denote by $E_{i j}$ the set of all edges $e \in E$ such that one end of $e$ is in $V_{i}$ and the other is in $V_{j}$ and define $e_{i j}=\left|E_{i j}\right|$. For an arbitrary set of edges $E$ from $K(v)$, denote by $\langle E\rangle$ the subgraph edge-induced by $E$.

The following lemma was proved in [8].

Lemma $1\left[8\right.$, Lemma 1]. Let $u=\left(u_{1}, \ldots, u_{i}, \ldots, u_{j}, \ldots u_{t}\right) \rightarrow v=\left(\ldots, u_{i}-1, \ldots u_{j}+1, \ldots\right)$ be an elementary transformation of a partition $u$, where $u_{t} \geq 2$. Then the graphs $K(u)$ and $H$ are not chromatically equivalent.

It is clear that every complete $t$-partite graph is $t$-colorable, but is not $(t-1)$-colorable; in other words, the chromatic number of a complete $t$-partite graph is equal to $t$. Let us compute $p t(K(u))$ for a complete multipartite graph $K\left(u_{1}, u_{2}, \ldots, u_{t}\right)$. It is easy to show that any partition of the vertex set of the graph $K(u)$ into $t+1$ parts can be obtained by splitting exactly one part into two nonempty subsets; so $p t(K(u))=\sum_{i=1}^{n} 2^{u_{i}-1}-t$.

It was investigated in [4] how the invariant $p t$ changes from the graph $K(v)$ to the graph $H$. Let us introduce all necessary definitions and auxiliary statements.

A complete multipartite subgraph $G_{1}$ of a graph $K(v)$ is called an E-subgraph if each part of the graph $G_{1}$ is contained in some part of the graph $K(v)$, and the edge set of the graph $G_{1}$ is contained in the set $E$. An arbitrary disjoint set of $E$-subgraphs is called a garland. We will say that a garland $G^{\prime}$ destroys a part $V_{i}$ if every vertex of $V_{i}$ is contained in some $E$-subgraph of the garland $G^{\prime}$. A garland of cardinality $p$ which destroys exactly $p-1$ parts is called interesting. The set of all edges of all $E$-subgraphs of a garland is called the edge aggregate and is denoted by $E(G)$. The set of all vertices of all $E$-subgraphs of a garland is called the vertex aggregate and is denoted by $V(G)$. A garland is called $k$-edge if its edge aggregate contains exactly $k$ edges. The following properties were proved in [4].

(1) If the chromatic number of a graph $H$ is equal to $t$ and $p t(H)=1$, then every garland of cardinality $p$ destroys at most $p-1$ parts.

(2) Every garland is uniquely defined by its edge aggregate.

(3) $\Delta p t(H, K(v))$ is equal to the number of all interesting garlands.

The next lemma follows from these properties.

Lemma 2 [4, Corollary 2]. If a graph $H$ is obtained from a graph $K(v)$ by removing some set of edges $E$ and the graphs $K(u)$ and $H$ are chromatically equivalent, then $|E| \leq \Delta p t(H, K(v)) \leq 2^{|E|-1}$.

An improvement of this estimate was obtained in [10]. A subgraph $H^{\prime}$ of a graph $\langle E\rangle$ is called a coordinated subgraph of type $K(s, 1)$ if $H^{\prime} \simeq K(s, 1)$ and all $s$ vertices of degree 1 are in the same part of $K(v)$. A part $V_{j}$ of a graph $K(v)$ is called active if there exist a vertex $x \in V_{j}$ and an edge $e \in E$ such that $x$ and $e$ are incident. 
Lemma 3 [10, Theorem]. Let every active part of a graph $K(v)$ contain at least three vertices. If $E$ induces a coordinated subgraph of type $K(|E|, 1)$, then $\Delta p t(G, K(v))=2^{|E|}-1$; otherwise, $\Delta p t(G, K(v)) \leqslant 2^{|E|-1}+1$.

Let $G^{\prime}=\left\{G_{1}^{\prime}, G_{2}^{\prime}, \ldots, G_{p}^{\prime}\right\}$ be a garland. We will say that the garland $G^{\prime}$ is of type $H_{1} \dot{\cup} H_{2} \dot{\cup} \ldots \dot{\cup} H_{p}$, where $\left\{H_{1}, H_{2}, \ldots H_{p}\right\}$ is a set of graphs, if $G_{i}^{\prime} \simeq H_{i}$ for all $i=1,2, \ldots, p$. We will say that an edge set $F \subseteq E$ induces a garland (an interesting garland) if there exists a garland (an interesting garland, respectively) $G$ such that $F=E(G)$. A set $F \subseteq E$ is called continuable if there exists a garland $G$ such that $F \subseteq E(G)$. A set $F \subseteq E^{\prime} \subseteq E$ is called continuable outside $E^{\prime}$ if there exists a garland $G$ such that $E(G) \cap E^{\prime}=F$. We will say that an edge $e$ is in the garland $G$ if $e \in E(G)$.

Let $e$ be an arbitrary edge from $E$. Denote by $\xi_{1}(e)$ the number of triangles of the graph $K(v)$ containing the edge $e$. Let $\xi_{1}=\sum_{e \in E} \xi_{1}(e)$.

Consider a triangle in a graph $G$ that contains exactly two edges from $E$. Denote them by $e_{1}$ and $e_{2}$. We will call a subgraph induced by $\left\{e_{1}, e_{2}\right\}$ a $\Xi_{2}$-subgraph. Denote by $\xi_{2}$ the number of such subgraphs and by $\xi_{3}$ the number of triangles in $\langle E\rangle$.

Denote by $I_{3}(G)$ the number of triangles in the graph $G$. In [2], the following equality was established:

$$
\Delta I_{3}(K(v), H)=\xi_{1}-\xi_{2}-2 \xi_{3} .
$$

Note that, the removal of an edge cannot produce a new triangle; so $\Delta I_{3}(K(v), H)$ is equal to the number of triangles in $K(v)$ destroyed by removing an edge set $E$ from $K(v)$.

\section{Upper bound for the invariant $p t$ in the case where $\langle E\rangle$ contains a triangle}

The main goal of this section is, in the case where $\langle E\rangle$ contains a triangle, to obtain an upper bound for the number of interesting garlands better than the bound in Lemma 3. Denote by $\Delta$ the edge set of this triangle. One the way to achieve this is to count number of garlands whose edge aggregates contain an edge from $\Delta$ and an edge that is not in the triangle. The following lemma was proved in [10].

Lemma 4 [10, Lemma 3]. If a set of edges $\left\{e_{1}, e_{2}, e_{3}\right\}=E_{1} \subset E$ induces a triangle, then there is no nonempty set $\hat{E} \subseteq E \backslash E_{1}$ such that the sets $\left\{e_{i}\right\} \dot{\cup} E_{1}$ induce interesting garlands for all $i=1,2,3$.

We can deduce the following statement from this lemma.

Corollary 1. If $\Delta$ induces a triangle, $\Delta \subseteq E$, and $F$ is an arbitrary nonempty subset of $E \backslash \Delta$, then there exist at most three nonempty subsets $F^{\prime} \subseteq \Delta$ such that $F \cup F^{\prime}$ induces an interesting garland.

$\mathrm{P}$ r o o f. Let us fix some $F \subseteq E \backslash \Delta$ and consider all possible nonempty subsets $F^{\prime} \subseteq \Delta$. Define $\Delta=\left\{e_{1}, e_{2}, e_{3}\right\}$. Assume that $F \cup F^{\prime}$ induces an interesting garland $G^{\prime}$.

Assume that $\left|F^{\prime}\right|=2$ and, without loss of generality, let $F^{\prime}=\left\{e_{1}, e_{2}\right\}$. Define $e_{1}=x y$ and $e_{2}=y z$. Note that the vertices $x$ and $z$ are in different parts of $K(v)$. Since $F \cup F^{\prime}$ induces a garland and the edges $e_{1}$ and $e_{2}$ are adjacent, they are in the same $E$-subgraph of $G^{\prime}$. Denote this $E$-subgraph by $H_{1}$. Since $H_{1}$ is a complete multipartite graph and the vertices $x$ and $z$ are in different parts, the edge $x z$ is in the edge aggregate of $G^{\prime}$. However, $x z \notin F^{\prime}$ and $x z \in \Delta$; therefore, $x z \notin F$. Consequently, $x z$ is not in the edge aggregate of $G^{\prime}$, a contradiction. Hence, $\left|F^{\prime}\right| \neq 2$. 
Thus, there are four choices for $F^{\prime}$ : three one-element subsets and $\Delta$. Using Lemma 4 , one can deduce that at most two of the sets $F^{\prime} \cup\left\{e_{1}\right\}, F^{\prime} \cup\left\{e_{2}\right\}$, and $F^{\prime} \cup\left\{e_{3}\right\}$ induce an interesting garland. Thus, there are at most three choices for $F^{\prime}$ and the corollary is proved.

The following lemma describes all sets $F \subseteq E \backslash \Delta$ such that there exist exactly three subsets $F^{\prime} \subseteq\left\{e_{1}, e_{2}, e_{3}\right\}$ such that $F \cup F^{\prime}$ induces an interesting garland.

Lemma 5. Assume that each part of $K(v)$ contains at least three vertices and edges $e_{1}, e_{2}, e_{3} \in E$ induce a triangle. Let $F \subseteq E \backslash\left\{e_{1}, e_{2}, e_{3}\right\}$. If there are exactly three nonempty subsets $F^{\prime} \subseteq\left\{e_{1}, e_{2}, e_{3}\right\}$ such that $F \cup F^{\prime}$ induces an interesting garland, then $F$ induces an interesting garland, $|F|>1$, and there is no edge $f \in F$ such that $f$ is adjacent to any of $e_{1}, e_{2}$, and $e_{3}$.

P r o o f. Define $\Delta=\left\{e_{1}, e_{2}, e_{3}\right\}$. Fix some $F \subseteq E \backslash \Delta$ and assume that there exist three different subsets $F^{\prime} \subseteq \Delta$ such that $F \cup F^{\prime}$ induces an interesting garland. Denote this subsets by $E_{1}, E_{2}$, and $E_{3}$. Using Corollary 1, one can deduce that two of $E_{1}, E_{2}$, and $E_{3}$ are one-element sets and the third one is equal to $\Delta$. Without loss of generality, let $E_{1}=\left\{e_{1}\right\}, E_{2}=\left\{e_{2}\right\}$, and $E_{3}=\Delta$.

Let the set $E_{1} \cup F$ induce an interesting garland $G_{1}$, the set $E_{2} \cup F$ induce an interesting garland $G_{2}$, and the set $E_{3} \cup F$ induce an interesting garland $G_{\Delta}$. Denote the vertices of the triangle by $x, y$, and $z$.

Let us prove that an arbitrary edge $f \in F$ is not adjacent to any of the edges $e_{1}, e_{2}$, and $e_{3}$. By contradiction, assume that $f$ is incident to some vertex from the triangle, without loss of generality, assume that $f$ is incident to $x$. Denote the second end of $f$ by $w$. Note that $w$ and $y$ or $w$ and $z$ are in different parts of $K(v)$. Without loss of generality, assume that $w$ and $y$ are in different parts of $K(v)$. Since the edges $x y$ and $f=x w$ are adjacent and are in the same $E$-subgraph of the garland $G_{\Delta}$, and $w$ and $y$ are in different parts of $K(v)$, the edge $w y$ is in $F$. Therefore, since $w x$ and $w y$ are adjacent edges, they are in the same $E$-subgraph of the garland $G_{1}$. So, the edge $x y$ is in the same $E$-subgraph. Thus, $x y$ is in the edge aggregate of the garland $G_{1}$. By analogy, the edge $x y$ is in the aggregate of the garland $G_{2}$. But this is impossible since $x y \in \Delta$ and the edge aggregates of the garlands $G_{1}$ and $G_{2}$ cannot contain a common edge from $\Delta$.

Since any edge from $F$ is not adjacent to any edge from $\Delta$, the set $\left\{e_{1}\right\}$ induces a $E$-subgraph of the garland $G_{1}$. Therefore, the graph $\langle F\rangle$ consists of $E$-subgraphs, so $F$ induces a garland. Denote this garland by $G_{F}$.

Note that all the garlands $G_{1}, G_{2}$ and $G_{\Delta}$ have the same cardinality. Denote this cardinality by $p$. Since all this garlands are interesting, each of them destroys exactly $p-1$ parts of $K(v)$. Note also that $\left|G_{F}\right|=p-1$.

Let $x \in V_{i}, y \in V_{j}, z \in V_{k}, e_{1}=x y$, and $e_{2}=y z$. Note that $V\left(G_{\Delta}\right)=V\left(G_{1}\right) \cup V\left(G_{2}\right)$. This means that if $G_{1}$ or $G_{2}$ destroy some part, then $G_{\Delta}$ also destroys this part. Note also that if one of the garlands $G_{1}, G_{2}$, and $G_{\Delta}$ destroys some part $V$ other than $V_{i}, V_{j}$, and $V_{k}$, then $G_{F}$ also destroys $V$. Since no edge from $E\left(G_{1}\right)$ is incident to $z$, the garland $G_{1}$ cannot destroy $V_{k}$. If $G_{2}$ destroys $V_{k}$, then $G_{\Delta}$ also destroys $V_{k}$ and thus $G_{\Delta}$ destroys more parts than $G_{1}$ does, a contradiction. Therefore, $G_{2}$ does not destroy $V_{k}$. If $G_{2}$ does not destroy $V_{j}$, then $G_{F}$ destroys $p-1$ parts and $\left|G_{F}\right|=p-1$, a contradiction. So $G_{2}$ destroys $V_{j}$. Since no edge from $F$ is incident to $y$, the garland $G_{F}$ cannot destroy $V_{j}$, thus $G_{F}$ destroys one part less than $G_{2}$ does. Therefore, $G_{F}$ destroys $p-1$ parts, and $G_{F}$ is an interesting garland.

It remains to prove that $|F|>1$. If $|F|=1$, then $G_{\Delta}$ is a garland of type $K(1,1,1) \dot{\cup} K(1,1)$. Note that such a garland cannot destroy any part, since each active part contains at least three vertices.

Now we are ready to prove a better upper bound in the case where $\langle E\rangle$ contains a triangle. 
Lemma 6. Assume that each part of $K(v)$ contains at least three vertices. If $\langle E\rangle$ contains a triangle, then the number of interesting garland does not exceed $2^{|E|-2}+2^{|E|-3}-3|E|+13$.

P r o o f. Denote edges of the triangle by $e_{1}, e_{2}$, and $e_{3}$, and let $\Delta=\left\{e_{1}, e_{2}, e_{3}\right\}$. Define $E^{\prime}=E \backslash \Delta$.

Case 1. Assume that $E^{\prime}$ induces a coordinated subgraph of type $K(|E|-3,1)$. Then all edges of $E$ are located in one of the ways shown in Fig. 1.
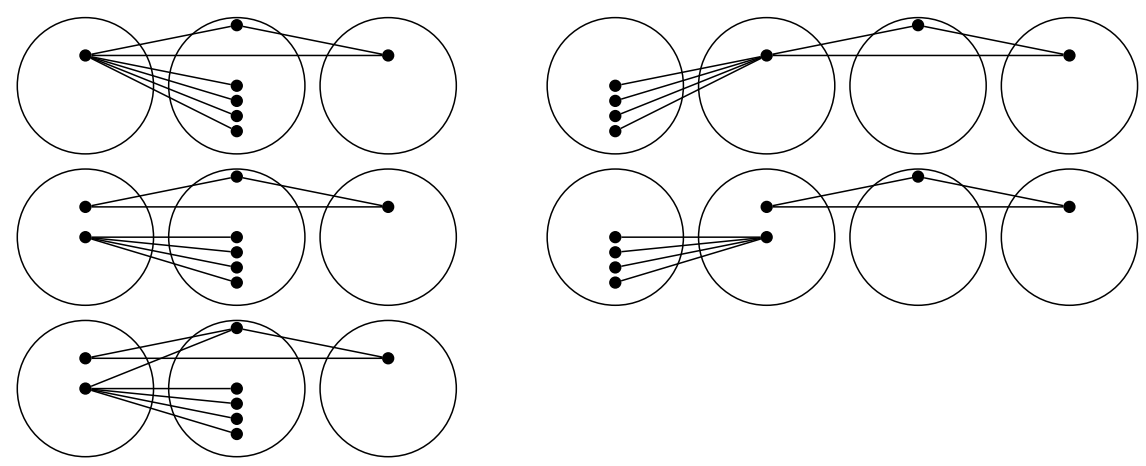

Figure 1. Triangle and a coordinated subgraph of type $K(|E|-3,1)$.

In the first case, there are $2+1+2^{|E|-2}-1=2^{|E|-2}+2$ interesting garlands.

In the second case, there are $4+2^{|E|-3}-1=2^{|E|-3}+3$ interesting garlands.

In the third case, there are at most $4+2^{|E|-3}-1+3=2^{|E|-3}+6$ interesting garlands.

In the fourth case, there are $4+2^{|E|-3}-1=2^{|E|-3}+3$ interesting garlands.

In the fifth case, there are at most $4+2^{|E|-3}-1+1=2^{|E|-3}+4$ interesting garlands.

In the sixth case, there are at most $2^{|E|-3}+6<2^{|E|-2}+2^{|E|-3}-3|E|+13$ interesting garlands.

In all the cases, the number of interesting garlands does not exceed $2^{|E|-3}+6<2^{|E|-2}+2^{|E|-3}-$ $3|E|+13$.

Case 2. Now consider the case where $E^{\prime}$ does not induce a coordinated subgraph of type $K(|E|-3,1)$.

There are four interesting garlands $G$ such that $E(G) \subseteq \Delta$. By Lemma 3, there exist at most $2^{|E|-4}+1$ interesting garlands $G$ such that $E(G) \subseteq E^{\prime}$.

Estimate the number of interesting garlands whose edge aggregates contain some edge from $E^{\prime}$ and some edge from $\Delta$. Define $F=E(G), F_{\Delta}=F \cap \Delta$, and $F^{\prime}=F \cap E^{\prime}$. By Lemma 5 , if for $F$ there exist three $F^{\prime}$ such that $F \cup F^{\prime}$ induces an interesting garland, then $F^{\prime}$ induce an interesting garland, $\left|F^{\prime}\right|>1$, and any edge from $F^{\prime}$ is not adjacent to any edge from $\Delta$. Denote the number of such $F$ by $X$.

Case 2.1. Assume that any edge from $E^{\prime}$ is not adjacent to any edge from $\Delta$. Using Lemma 3 and the fact that $|F|>1$, one can deduce that

$$
X \leqslant 2^{|E|-4}+1-(|E|-3)=2^{|E|-4}-|E|+4 .
$$

For any other $F^{\prime} \subseteq E^{\prime},\left|F^{\prime}\right|>1$, there are at most two subsets $F_{\Delta} \subseteq \Delta$ such that $F^{\prime} \cup F_{\Delta}$ induces an interesting garland. If $F^{\prime} \subseteq E^{\prime}$ and $\left|F^{\prime}\right|=1$, there exist no $F_{\Delta} \subseteq \Delta$ such that $F^{\prime} \cup F_{\Delta}$ induces 
an interesting garland. Therefore, the number of interesting garlands does not exceed

$$
\begin{gathered}
3 X+2\left(2^{|E|-3}-1-X-(|E|-3)\right)+2^{|E|-4}+1+4= \\
=3 X+2^{|E|-2}-2-2 X-2|E|+6+2^{|E|-4}+1+4= \\
=2^{|E|-2}+X-2|E|+2^{|E|-4}+9 \leqslant \\
\leqslant 2^{|E|-2}+2^{|E|-4}-|E|+4-2|E|+2^{|E|-4}+9=2^{|E|-2}+2^{|E|-3}-3|E|+13 .
\end{gathered}
$$

Case 2.2. Now consider the case when there are exactly $k \geqslant 1$ edges from $E^{\prime}$ such that each of them is adjacent to some edge from $\Delta$. Denote the set of this edges by $H$.

If for a set $F^{\prime} \subseteq E^{\prime}$ there exists exactly three sets $F_{\Delta} \subseteq \Delta$ such that $F^{\prime} \cup F_{\Delta}$ induces an interesting garland, then $F^{\prime} \subseteq E^{\prime} \backslash H$ and $\left|F^{\prime}\right|>1$, so

$$
X \leqslant 2^{|E|-3-k}-(|E|-3-k)-1 .
$$

For any other $F^{\prime} \subseteq E^{\prime},\left|F^{\prime}\right|>1$, there are at most two such sets $F_{\Delta}$. If $\left|F^{\prime}\right|=1$, then if $F^{\prime} \subseteq H$, then there is at most one such $F_{\Delta}$ and if $F^{\prime} \nsubseteq \subset H$, then such $F_{\Delta}$ does not exist. Therefore, the number of interesting garland does not exceed

$$
\begin{gathered}
3 X+2\left(2^{|E|-3}-1-X-(|E|-3)\right)+k+2^{|E|-4}+1+4= \\
3 X+2^{|E|-2}-2-2 X-2|E|+6+2^{|E|-4}+1+4+k= \\
=2^{|E|-2}+X-2|E|+2^{|E|-4}+9+k \leqslant \\
\leqslant 2^{|E|-2}-2|E|+2^{|E|-4}+9+2^{|E|-3-k}-(|E|-3-k)-1+k= \\
=2^{|E|-2}+2^{|E|-4}-3|E|+11+2^{|E|-3-k}+2 k .
\end{gathered}
$$

It remains to prove that

$$
2^{|E|-2}+2^{|E|-4}-3|E|+11+2^{|E|-3-k}+2 k \leqslant 2^{|E|-2}+2^{|E|-3}-3|E|+13 .
$$

It is sufficient to prove that

$$
2^{|E|-3-k}+2 k \leqslant 2^{|E|-3}+2 .
$$

Let $m=|E|-3$. Consider the function $f(x)=2^{m-x}+2 x$ defined on the closed interval from 1 to $m$. Calculate the first and second derivatives:

$$
\begin{aligned}
& f^{\prime}(x)=-2^{m-x} \ln 2+2 ; \\
& f^{\prime \prime}(x)=2^{m-x} \ln ^{2} 2>0 .
\end{aligned}
$$

Therefore, $f$ is a convex function, so it takes its maximum value at the end point of the interval. So it suffices to verify the inequality for $k=1$ and $k=m=|E|-3$. For $k=1$, the inequality takes the form

$$
2^{|E|-3}+2 \leqslant 2^{|E|-3}+2
$$

and is valid. Consider the case $k=m=|E|-3$. In this case, we need to prove that $2 m \leqslant 2^{m}+2$. Dividing both sides by 2 , one can obtain $m-1 \leqslant 2^{m-1}$. By Bernulli's inequality,

$$
2^{m-1}=(1+1)^{m-1} \geqslant 1+m-1=m>m-1,
$$

and the lemma is proved.

In some cases, we can use a better upper bound than Lemma 3 gives. In such cases, the following lemma is useful. 
Lemma 7. Assume that each part of $K(v)$ contains at least three vertices and edges $e_{1}, e_{2}, e_{3} \in E$ induce a triangle. Let $E^{\prime}=E \backslash\left\{e_{1}, e_{2}, e_{3}\right\}$. If there are $X$ interesting garlands $G$ such that $E(G) \subseteq E^{\prime}, Y$ nonempty subsets of $E^{\prime}$ continuable outside $E^{\prime}, k$ edges $h \in E^{\prime}$ for each of which there exists an edge $h^{\prime} \in \Delta$ such that $h$ and $h^{\prime}$ induce a coordinated subgraph of type $K(2,1)$, then the number of interesting garlands doest not exceed $2 X+2 Y-5|E|+19+k$.

$\mathrm{P}$ r o o f. Consider an arbitrary subset $F \subseteq E^{\prime}$ and estimate the number of interesting garlands $G$ such that $F \subseteq E(G)$ and $E(F) \cap\left\{e_{1}, e_{2}, e_{3}\right\} \neq \varnothing$. Let $H=E(F) \cap\left\{e_{1}, e_{2}, e_{3}\right\}$. Note that $F$ is a nonempty subset of $E^{\prime}$ continuable outside $E^{\prime}$.

Case 1. $|F|=1$. Let $F=\{f\}$. If $f$ does not induce a coordinated subgraph of type $K(2,1)$ with any of edges from $\left\{e_{1}, e_{2}, e_{3}\right\}$, then $H$ must be an $E$-subgraph. This means that either $H$ is a one-element set or $H=\left\{e_{1}, e_{2}, e_{3}\right\}$. In both cases, $F \cup H$ does not induce an interesting garland. Therefore, $f$ must induce a coordinated subgraph of type $K(2,1)$ with some edge from $\left\{e_{1}, e_{2}, e_{3}\right\}$. By the lemma hypothesis, there are $k$ such edges. Note that, in this case, $H$ must be a one-element set and it is uniquely defined by the edge $f$. So, in this case, there are at most $k$ interesting garlands.

Case 2. $|F|>1$ and $F$ does not induce interesting garlands. By Lemma 5, there are at most two subsets $H$. Note that $F$ can be chosen in

$$
Y-X-(|E|-3)=Y-X-|E|+3
$$

ways; so, in this case, the number of interesting garlands does not exceed

$$
2(Y-X-|E|+3)=2 Y-2 X-2|E|+6 .
$$

Case 3. $|F|>1$ and $F$ induces interesting garlands. In this case, by Lemma 7, there are at most three sets $H$, so the number of interesting garlands does not exceed

$$
3(X-(|E|-3))=3 X-3|E|+9 .
$$

There are $X$ interesting garlands whose edge aggregates are in the set $E^{\prime}$ and four interesting garlands whose edge aggregates are in the set $\left\{e_{1}, e_{2}, e_{3}\right\}$. Therefore, the number of interesting garlands does not exceed

$$
2 Y-2 X-2|E|+6+3 X-3|E|+9+k+X+4=2 Y+2 X-5|E|+19,
$$

and the lemma is proved.

Lemma 8. Assume that each active part of $K(v)$ contains at least three vertices. If $\langle E\rangle$ contains two triangles that do not have a common edge, then the number of interesting garlands does not exceed $2^{|E|-2}-12|E|+58$.

P r o o f. Denote by $\Delta$ the edge set of one triangle, and let $E^{\prime}=E \backslash \Delta$. Let $X$ be the number of interesting garlands $G$ such that $E(G) \subseteq E^{\prime}$, and let $Y$ be the number of nonempty subsets of $E^{\prime}$ continuable outside $E^{\prime}$.

By Lemma 6,

$$
X \leqslant 2^{|E|-5}+2^{|E|-6}-3(|E|-3)+13=3 \cdot 2^{|E|-6}-3|E|+22 .
$$

Denote by $\Delta^{\prime}$ the edge set of the other triangle. To estimate the number of nonempty subsets of $E^{\prime}$ continuable outside $E^{\prime}$, consider an arbitrary $F \subseteq E^{\prime} \backslash \Delta^{\prime}$ and estimate the number of sets 
$F^{\prime} \subseteq \Delta^{\prime}$ such that $F \cup F^{\prime}$ is a continuable set. Note that if $F \cup F^{\prime}$ is a continuable set, then $F^{\prime}$ is either empty, or a one-element set, or $\Delta^{\prime}$. Since $F$ can be chosen in $2^{|E|-6}$ ways, there are at most $5 \cdot 2^{|E|-6}$ continuable sets; thus, there are at most $5 \cdot 2^{|E|-6}$ nonempty continuable sets.

Using Lemma 7 and assuming $k \leqslant|E|-3$, one can conclude that the number of interesting garlands does not exceed

$$
\begin{gathered}
2 \cdot\left(3 \cdot 2^{|E|-6}-3|E|+22\right)+2 \cdot\left(5 \cdot 2^{|E|-6}-1\right)-5|E|+19+|E|-3= \\
=16 \cdot 2^{|E|-6}-12|E|+55=2^{|E|-2}-12|E|+58 .
\end{gathered}
$$

\section{Upper bound for the invariant $p t$ in the case where $\langle E\rangle$ contains a $\Xi_{2}$-subgraph}

The main goal of this section is, in the case where $\langle E\rangle$ contains a $\Xi_{2}$-subgraph, to obtain a better upper bound for the number of interesting garlands. Recall that a pair of edges $e, f \in E$ induces a $\Xi_{2}$-subgraph if it induces a triangle in $K(v)$ whose third edge is not in $E$.

Lemma 9. If edges $f$ and $g$ induce a $\Xi_{2}$-subgraph, then there is no a garland $G$ such that $f, g \in E(G)$.

P r o o f. Define $f=x y$ and $g=y z$ and note that the vertices $x$ and $z$ are in different parts of $K(v)$.

By contradiction, assume that there is a garland $G$ such that $f, g \in E(G)$. The edges $f$ and $g$ are adjacent, so they are in the same $E$-subgraph $H^{\prime}$ of the garland $G$. Since the vertices $y$ and $z$ are in different parts of $K(v)$, the edge $y z$ must be in the subgraph $H^{\prime}$, so $y z \in E$, a contradiction.

Now we are ready to prove a better upper bound.

Lemma 10. Let $|E| \geqslant 4$ and each active part of $K(v)$ contains at least three vertices. If $\langle E\rangle$ contains a $\Xi_{2}$-subgraph, then the number of interesting garlands does not exceed $2^{|E|-1}$.

P r o o f. Let edges $f$ and $g$ induce a $\Xi_{2}$-subgraph. Consider the cases.

Case 1. Assume that $\langle E\rangle$ contains a coordinated subgraph of type $K(|E|-1,1)$. Without loss of generality, assume that $f$ is not in the coordinated subgraph of type $K(|E|-1,1)$. Then there are two possible configurations for $E$, they are shown in Fig. 2.
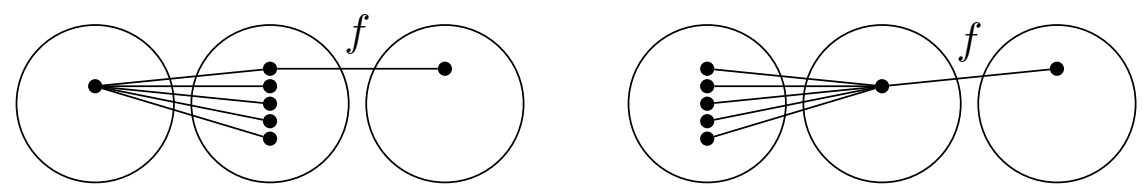

Figure 2. $\Xi_{2}$-subgraph and coordinated subgraph of type $K(|E|-1,1)$.

In both cases, the edge $f$ is in exactly one interesting garland. There are exactly $2^{|E|-1}-1$ interesting garlands that do not contain the edge $f$. Thus, there are exactly

$$
2^{|E|-1}-1+1=2^{|E|-1}
$$


interesting garlands.

Case 2. Now consider the case where $\langle E\rangle$ does not contain a coordinated subgraph of type $K(|E|-1,1)$. By Lemma 9, the edge aggregate of any garland is in the set $E \backslash\{f\}$ or in the set $E \backslash\{e\}$. By Lemma 3, there are at most $2^{|E|-2}+1$ interesting garlands whose edge aggregates are in the set $E \backslash\{f\}$ and at most $2^{|E|-2}+1$ interesting garlands whose edge aggregates are in the set $E \backslash\{e\}$. Note that $|E|-2$ interesting one-edge garlands were counted twice, so the number of interesting garlands does not exceed

$$
2 \cdot\left(2^{|E|-2+1}\right)-(|E|-2)=2^{|E|-1}-|E|+4 \leqslant 2^{|E|-1},
$$

and the lemma is proved.

In the case where $\langle E\rangle$ does not contain a coordinated subgraph of type $K(|E|-1,1)$, we can obtain a better upper bound.

Lemma 11. Let $|E| \geq 5$, and let each part of $K(v)$ contain at least three vertices. If $\langle E\rangle$ does not contain a coordinated subgraph of type $K(|E|-1,1)$ and contains $a \Xi_{2}$-subgraph, then the number of interesting garlands does not exceed $2^{|E|-1}-|E|+1$.

P r o o f. By Lemma 6, it is sufficient to consider the case where $\langle E\rangle$ does not contain a triangle.

Let edges $e$ and $g$ induce a $\Xi_{2}$-subgraph. Note that, by Lemma 9 , the edge aggregate of any garland cannot contain both the edges $e$ and $g$.

Case 1. Assume that the edge $g$ is in two different $\Xi_{2}$-subgraphs. Define $E_{e}=E \backslash\{g\}$ and note that if $e \in E(G)$, then $E(G) \subseteq E_{e}$. By the lemma hypothesis, $E_{e}$ does not induce a coordinated subgraph of type $K(|E|-1,1)$, so, by Lemma 3, there are at most $2^{|E|-2}+1$ interesting garlands whose edge aggregates are subsets of $E_{e}$. In this case, it remains to estimate the number of interesting garlands whose edge aggregates contain the edge $g$.

Let $f \neq e$ be an edge such that the edges $f$ and $g$ induce a $\Xi_{2}$-subgraph. Define $E_{g}=E \backslash\{f, e\}$ and note that if the edge $g$ is in $G$, then $E(G) \subseteq E_{g}$.

Case 1.1. Assume that $E_{g}$ induces a coordinated subgraph of type $K\left(\left|E_{g}\right|, 1\right)$. Then there are exactly $2^{|E|-2}-1$ interesting garlands whose edge aggregates are subsets of $E_{g}$. Recall that there are at most $2^{|E|-2}+1$ interesting garlands whose edge aggregates are subsets of $E_{e}$. Note also that there are $2^{|E|-3}$ interesting garlands whose edge aggregates are subsets of $E \backslash\{e, g\}$ (which are $2^{|E|-3}-1$ interesting garlands of type $K(s, 1)$ whose edge aggregates are subsets of $E \backslash\{e, g, f\}$ and a one-edge garland induced by $f$ ). All these garlands were counted twice, so the number of interesting garlands does not exceed

$$
2^{|E|-2}-1+2^{|E|-2}+1-2^{|E|-3}=2^{|E|-1}-2^{|E|-3} .
$$

It remains to prove that

$$
2^{|E|-1}-2^{|E|-3} \leqslant 2^{|E|-1}-|E|+1 .
$$

To do this, it suffices to prove that the inequality $n-1 \leqslant 2^{n-3}$ holds for all integer $n \geqslant 5$. Let us prove this by induction on $n$.

The base case. If $n=5$, then $5-1=4=2^{2}$ and the inequality is verified.

The induction step. Assume that the inequality is proved for $n$ and prove it for $n+1$. We need to prove $2^{n-2} \geqslant n$. By the induction hypothesis,

$$
2^{n-2}=2 \cdot 2^{n-3} \geqslant 2 \cdot(n-1)=n+n-2 \geqslant n+3>n,
$$


and the inequality is proved.

Case 1.2. Now consider the case where $E_{g}$ does not induce a coordinated subgraph of type $K\left(\left|E_{g}\right|, 1\right)$. In this case, by Lemma 3 , there are at most $2^{|E|-3}+1$ interesting garlands whose edge aggregates are subsets of $E_{g}$. Note also that there are $|E|-3$ interesting one-edge garlands induced by edges from $E \backslash\{e, g\}$. So, the number of interesting garlands does not exceed

$$
2^{|E|-3}+1+2^{|E|-2}+1-(|E|-3)=3 \cdot 2^{|E|-3}+5 .
$$

It remains to prove that

$$
3 \cdot 2^{|E|-3}-|E|+5 \leqslant 2^{|E|-1}-|E|+1 .
$$

It suffices to check that

$$
3 \cdot 2^{|E|-3}+4 \leqslant 2^{|E|-1}=4 \cdot 2^{|E|-3},
$$

which is equivalent to $4 \leqslant 2^{|E|-3}$, which is true because $|E| \geqslant 5$.

Case 2. Now consider the case where the edges $f$ and $g$ are in only one $\Xi_{2}$-subgraph. Define $E_{g}=E \backslash\{e\}$ and $E_{e}=E \backslash\{e\}$. Note that, by Lemma 3, there are at most $2^{|E|-1}+1$ interesting garlands whose edge aggregates are subsets of $E_{g}$ and at most $2^{|E|-1}+1$ interesting garlands whose edge aggregates are subsets of $E_{e}$.

Assume that there exist at least $|E|+1$ interesting garlands whose edge aggregates are subsets of $E \backslash\{e, g\}$. In this case, there are at most

$$
2\left(2^{|E|-1}+1\right)-(|E|+1)=2^{|E|}+2-|E|-1=2^{|E|}-|E|+1
$$

interesting garlands. Therefore, it suffices to prove the lemma in the case when there are at most $|E|$ garlands whose edge aggregates are subsets of $E \backslash\{e, g\}$.

Note that, in any case, there are $|E|-2$ interesting one-edge garlands induced by edges from $E \backslash\{e, g\}$. Consequently, there are at most two interesting garlands whose edge aggregates are subsets of $E \backslash\{e, g\}$ and contain at least two edges.

Note that it suffices to prove that the edge $f$ is in at most $2^{|E|-2}-|E|$ interesting garlands. Indeed, in this case, since there are at most $2^{|E|-1}+1$ interesting garland whose edge aggregates are subsets of $E_{g}$, the total number of interesting garlands does not exceed

$$
2^{|E|-2}-|E|+2^{|E|-2}+1=2^{|E|-1}-|E|+1 .
$$

By analogy, it suffices to prove that the edge $g$ is in at most $2^{|E|-2}-|E|$ interesting garlands.

To prove this, we build either $|E|$ subsets $F \subseteq E \backslash\{e, f\}$ such that $F \cup\{f\}$ does not induce an interesting garland or $|E|$ subsets $F \subseteq E \backslash\{e, f\}$ such that $F \cup\{g\}$ does not induce an interesting garland.

Case 2.1. Assume that the edge $e$ is in three different coordinated subgraphs of type $K(2,1)$. In this case, either $E \backslash\{e, g\}$ contains a coordinated subgraph of type $K(3,1)$ and so contains at least three interesting garlands with more than one edge or $g$ is in two different $\Xi_{2}$-subgraphs. Both cases are impossible.

Case 2.2. Assume that the edge $e$ is in two different coordinated subgraphs of type $K(2,1)$. Let edges $h_{1}$ and $h_{2}$ be such that the pairs of edges $\left\{e, h_{1}\right\}$ and $\left\{e, h_{2}\right\}$ induce $\Xi_{2}$-subgraphs. Note that, in this case, the edge $e$ is in the coordinated subgraph of type $K(3,1)$ because, otherwise, the edge $g$ is in two $\Xi_{2}$-subgraphs.

Consider an arbitrary edge $\hat{h} \in E \backslash\left\{e, g, h_{1}, h_{2}\right\}$. Note that the set $\{e, \hat{h}\}$ does not induce a coordinated subgraph of type $K(2,1)$; the set $\left\{e, h_{1}, \hat{h}\right\}$ does not induce an interesting garland, since an interesting three-edge garland must be of type $K(3,1)$, a triangle, or $K(2,1) \cup(1,1)$, and 
all cases are impossible. By analogy, the set $\left\{e, h_{2}, \hat{h}\right\}$ does not induce an interesting garland. So, we have built

$$
3 \cdot(|E|-4)=3|E|-12=|E|+2(|E|-6)
$$

necessary subsets. If $|E| \geqslant 6$, then the lemma is proved.

Consider the case $|E|=5$. Denote by $g^{\prime}$ a single edge from $E \backslash\left\{e, g, h_{1}, h_{2}\right\}$. Note, that the sets $\left\{g, h_{1}\right\},\left\{g, h_{2}\right\},\left\{g, h_{1}, h_{2}\right\},\left\{g, g^{\prime}, h_{1}\right\}$, and $\left\{g, g^{\prime}, h_{2}\right\}$ do not induce interesting garlands (see Fig. 3 ) and, in this case, the lemma is proved.

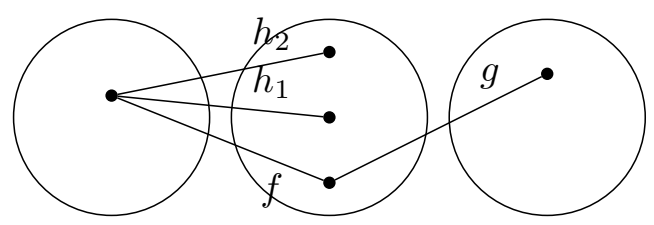

Figure 3. Cordinated subgraph of type $K(3,1)$ and $\Xi_{2}$-subgraph.

Case 2.3. Assume that the edge $e$ is in exactly one coordinated subgraph of type $K(2,1)$. Denote the second edge of this subgraph by $h$.

Case 2.3.1. Assume that the edge $g$ is not in any coordinated subgraph of type $K(2,1)$. Consider an arbitrary edge $f \in E \backslash\{e, g\}$. Note that the pair of edges $\{g, f\}$ does not induce a coordinated subgraph of type $K(2,1)$. Note also that, for an arbitrary edge $f \in E \backslash\{e, g, h\}$, the triple of edges $\{g, h, f\}$ does not induce an interesting garland. Therefore, we have built

$$
|E|-2+|E|-3=2|E|-5 \geqslant|E|
$$

necessary subsets for the edge $g$.

Case 2.3.2. Assume that the edge $g$ is in the coordinated subgraph of type $K(2,1)$. By the previous cases, it suffices to consider the case where $g$ is in exactly one coordinated subgraph of type $K(2,1)$. Denote the second edge of this subgraph by $h^{\prime}$. Note that the edges $e, g, h$, and $h^{\prime}$ form one of the two configurations shown in Fig. 4.
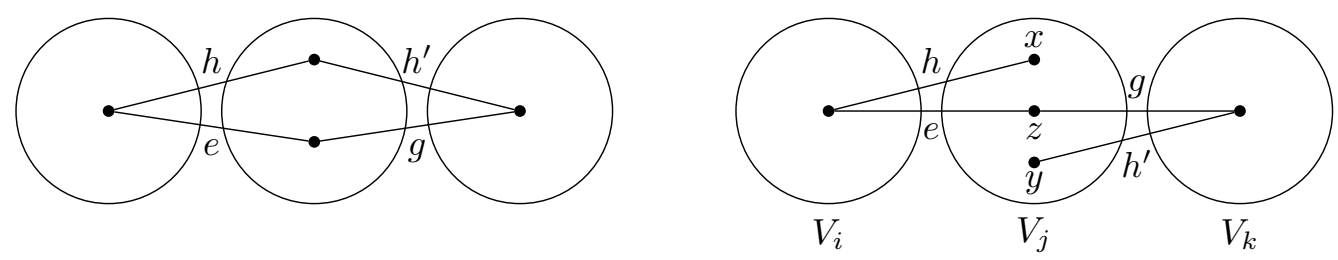

Figure $4 . \Xi_{2}$-subgraph and two coordinated subgraphs of type $K(2,1)$.

Consider an arbitrary edge $f \in E \backslash\left\{e, g, h^{\prime}\right\}$. Note that the pair of edges $\{g, f\}$ does not induce a coordinated subgraph of type $K(2,1)$. Note also that, for an arbitrary edge $f \in E \backslash\left\{e, g, h, h^{\prime}\right\}$, the triple of edges $\{g, h, f\}$ does not induce an interesting garland. Thus, we have built

$$
|E|-3+|E|-4=2|E|-7
$$

necessary subsets for the edge $g$. By analogy, we can build $2|E|-7$ necessary subsets for the edge $f$.

Let $\hat{h}$ be an arbitrary edge from $E \backslash\left\{e, g, h, h^{\prime}\right\}$. Consider the set $\left\{h, h^{\prime}, \hat{h}\right\}$. If the set $\left\{e, h, h^{\prime}, \hat{h}\right\}$ induces an interesting garland, this garland must be of type $K(2,1) \cup(2,1)$; the same is true for 
the set $\left\{g, h, h^{\prime}, \hat{h}\right\}$. Since both the sets $\{\hat{h}, h\}$ and $\left\{\hat{h}, h^{\prime}\right\}$ cannot induce a coordinated subgraph of type $K(2,1)$, at least one of the sets $\left\{e, h, h^{\prime}, \hat{h}\right\}$ and $\left\{g, h, h^{\prime}, \hat{h}\right\}$ does not induce an interesting garland. Therefore, we have built $2|E|-6$ necessary subsets for the edge $e$ or for the edge $g$, and the lemma is proved if $|E|>6$.

In the case where the edges $e, g, h$, and $h^{\prime}$ form the first configuration shown in Fig. 4, the sets $\left\{e, h, h^{\prime}\right\}$ and $\left\{g, h, h^{\prime}\right\}$ do not induce interesting garlands; consequently, we have built $2|E|-5$ necessary subsets for the edge $e$ or for the edge $g$.

It remains to prove the lemma in the case where $|E|=5$ and the edges $e, g, h$, and $h^{\prime}$ form the second configuration shown in Fig. 4. Note that there are at most eight interesting garlands whose edge aggregates are subsets of $\left\{g, f, h^{\prime}, h\right\}$ : four one-edge garlands, two garlands of type $K(2,1)$, and possibly two garlands of type $K(2,1) \dot{\cup} K(1,1)$. So it suffies to prove that there are at most five interesting garlands that contain the edge $\hat{h}$.

Case 2.3.2.1. Assume that the edge $\hat{h}$ is incident neither to the vertex $x$ nor the vertex $y$. In this case, the edge $\hat{h}$ is not in any coordinated subgraph of type $K(2,1)$ because, otherwise, either the edge $e$ is in two coordinated subgraphs of type $K(2,1)$ or the edge $e$ is in two coordinated subgraphs of type $K(2,1)$, both the cases lead to a contradiction. Therefore, the edge $\hat{h}$ is not in any two-edge garland. Moreover, since $\langle E\rangle$ does not contain a triangle, the edge $\hat{h}$ is not in any one-element garland except for the one-edge garland.

Assume that the edge $\hat{h}$ is in some garland $G$ of cardinality more than one. In this case, the garland $G$ must destroy some part. The vertex aggregate $V(G)$ has at most two common vertices with any part $V \neq V_{j}$. Thus, $V_{j}$ is the only part that can be destroyed by $G$. Note also that the edge $\hat{h}$ is not incident to the vertex $z$ because, otherwise, the edge $e$ or $g$ is in two $\Xi_{2}$-subgraphs. Therefore, either $e \in E(G)$ or $g \in E(G)$. Note also that this two edges cannot be in $E(G)$ simultaneously; thus, the edge $\hat{h}$ is in at most two garlands with cardinality more than one. Therefore, in this case, the edge $\hat{h}$ is in at most three garlands, and the lemma is proved in this case.

Case 2.3.2.2. Now assume that the edge $\hat{h}$ is incident either to the vertex $x$ or the vertex $y$. Without loss of generality, assume that $\hat{h}$ is incident to $x$. Note that, in this case, the edge aggregate that contains the edge $\hat{h}$ can be one of the following four sets: $\{\hat{h}\},\{\hat{h}, h\},\left\{\hat{h}, g, h^{\prime}\right\}$, and $\left\{\hat{h}, h, g, h^{\prime}\right\}$, and the lemma is proved in this case.

Case 2.4. It remains to consider the case where neither the edge $e$ nor the edge $g$ are in any coordinated subgraph of type $K(2,1)$. In this case, neither the set $\{e, f\}$ nor the set $\{g, f\}$ induce an interesting garland for any edge $f \in E \backslash\{e, g\}$, so we have built $|E|-2$ necessary subsets for the edges $e$ and $g$.

Since $|E \backslash\{e, g\}| \geqslant 3$, consider three arbitrary edges from this set and denote them by $h_{1}, h_{2}$ and $h_{3}$. Define $H=\left\{h_{1}, h_{2}, h_{3}\right\}$. Note that there exist exactly three two-element subsets $H^{\prime} \subseteq H$. If, for any two-element subset $H^{\prime} \subset H$, the set $H^{\prime} \cup\{e\}$ does not induce an interesting garland, then we have built $|E|+1$ necessary subsets for the edge $e$. So it suffices to consider the case where, for some two-element subset $H^{\prime} \subset H$, the set $H^{\prime} \cup\{e\}$ induces an interesting garland. Without loss of generality, $H^{\prime}=\left\{h_{1}, h_{2}\right\}$. Since $\langle E\rangle$ does not contain a triangle and the edge $e$ is not in any coordinated subgraph of type $K(2,1) S$, an interesting three-edge garland must be of type $K(2,1) \dot{\cup} K(1,1)$ or $K(1,1,1)$.

Case 2.4.1. The set $H^{\prime} \cup\{e\}$ induces an interesting garland of type $K(1,1,1)$. Note that, in this case, the set $\left\{g, h_{1}, h_{2}\right\}$ does not induce an interesting garland. Note also that at least one of the sets $\left\{g, h_{3}, h_{1}\right\}$ and $\left\{g, h_{3}, h_{2}\right\}$ does not induce an interesting garland. Therefore, we have built $|E|$ necessary subsets for the edge $g$, and, in this case, the lemma is proved.

Case 2.4.2. The set $H^{\prime} \cup\{e\}$ induces an interesting garland of type $K(2,1) \dot{\cup} K(1,1)$. Note 
that, in this case, sets $\left\{g, h_{3}, h_{1}\right\}$ and $\left\{g, h_{3}, h_{2}\right\}$ does not induce interesting garlands. Therefore, we have built $|E|$ necessary subsets for the edge $g$ and the lemma is proved.

Lemma 12. Assume that each active part of $K(v)$ contains at least three vertices. If an edge $e \in E$ is in $k \leqslant|E|-2$ different $\Xi_{2}$-subgraphs, then

(1) the edge $e$ is in at most $2^{|E|-k-1}$ interesting garlands;

(2) if $\langle E\rangle$ does not contain a coordinated subgraph of type $K(|E|-k, 1)$, then the edge $e$ is in at most $2^{|E|-k-1}-|E|+k+2$ interesting garlands.

P r o o f. Let edges $h_{1}, h_{2}, \ldots, h_{k}$ be such that the pair of edges $\left\{h_{i}, e\right\}$ induces a $\Xi_{2}$-subgraph for all $i=1,2, \ldots, k$. Define $E^{\prime}=E \backslash\left\{h_{1}, h_{2}, \ldots, h_{k}\right\}$.

Let $F$ be an edge aggregate of some interesting garland, and let $e \in F$. Note that $h_{i} \notin F$ for all $i=1,2, \ldots, k$. Therefore, $F \subseteq E^{\prime}$.

If $E^{\prime}$ does not induce a coordinated subgraph of type $K(|E|-k, 1)$, then, by Lemma 3, there exist at most $2^{|E|-k-1}$ interesting garlands whose edge aggregates are subsets of $E^{\prime}$. Note that among them there are $|E|-k-1$ one-edge garlands that do not contain the edge $e$. Consequently, the edge $e$ is in at most

$$
2^{|E|-k-1}+1-(|E|-k-1)=2^{|E|-k-1}-|E|+k+2
$$

interesting garland, and the lemma is proved.

Lemma 13. Assume that $|E| \geqslant 6$ and the subgraph $\langle E\rangle$ contains at least two $\Xi_{2}$-subgraphs and does not contain a coordinated subgraph of type $K(|E|-2)$.

(1) If there exists an edge, which is in two $\Xi_{2}$-subgraphs, then the number of interesting garlands does not exceed $2^{|E|-2}+2^{|E|-3}-|E|+5$.

(2) If there exist two $\Xi_{2}$-subgraphs without common edges, then the number of interesting garlands does not exceed $2^{|E|-1}-3|E|+6$.

P r o o f. Prove the first statement of the lemma. Assume that an edge $e$ is in two $\Xi_{2}$-subgraphs. Since $\langle E\rangle$ does not contain a coordinated subgraph of type $K(|E|-2)$, by Lemma 12, the edge $e$ is in at most $2^{|E|-3}-|E|+4$ interesting garlands. By Lemma 3, there are at most $2^{|E|-2}+1$ interesting garlands whose edge aggregates are subsets of $E \backslash\{e\}$. Consequently, there are at most

$$
2^{|E|-3}-|E|+4+2^{|E|-2}+1=2^{|E|-2}+2^{|E|-3}-|E|+5
$$

interesting garlands.

Now prove the second statement of the lemma. Assume that there are two $\Xi_{2}$-subgraphs without common edges. Let edges $e_{1}$ and $e_{2}$ induce a $\Xi_{2}$-subgraph. By Lemma 9 , the edge aggregate of any garland is a subset of $E \backslash\left\{e_{1}\right\}$ or $E \backslash\left\{e_{2}\right\}$. By Lemma 11, there are at most

$$
2^{|E|-2}-(|E|-1)+1=2^{|E|-2}-|E|+2
$$

interesting garlands whose edge aggregates are subsets of $E \backslash\left\{e_{1}\right\}$ and there are at most $2^{|E|-2}-$ $|E|+2$ interesting garlands whose edge aggregates are subsets of $E \backslash\left\{e_{2}\right\}$. Note that $|E|-2$ one-edge garlands were counted twice, so the number of interesting garlands does not exceed

$$
2\left(2^{|E|-2}-|E|+2\right)-(|E|-2)=2^{|E|-1}-3|E|+6,
$$

and the lemma is proved. 
Lemma 14. Assume that each active part of $K(v)$ contains at least three vertices and $\langle E\rangle$ contains a triangle. If an edge $e$ is in the triangle from $\langle E\rangle$ and is in $k$ different $\Xi_{2}$-subgraphs, then $e$ is in at most $2^{|E|-k-2}-|E|+k+3$ interesting garlands.

$\mathrm{P}$ r o o f. Let $\Delta$ be the edge set of a triangle from $\langle E\rangle$ that contains the edge $e$, and let edges $h_{1}, h_{2}, \ldots, h_{k}$ be such that the pairs of edges $\left\{e, h_{i}\right\}$ induce $\Xi_{2}$-subgraphs for all $i=1,2, \ldots k$. Define $E^{\prime}=E \backslash\left(\Delta \cup\left\{h_{1}, h_{2}, \ldots, h_{k}\right\}\right)$.

Let $G$ be an arbitrary garland such that $e \in E(G)$, and let $F=E(G)$. Note that $h_{i} \notin F$ for all $i=1,2, \ldots, k$. Define $F^{\prime}=F \cap E^{\prime}$. Note also that either $F \cap \Delta=\{e\}$ or $F \cap \Delta=\Delta$.

If $F \cap \Delta=\{e\}$, then $F^{\prime}$ can be chosen in $2^{|E|-k-3}$ ways. If $F \cap \Delta=\Delta$, then $\left|F^{\prime}\right|>1$ and, consequently, $F^{\prime}$ can be chosen in

$$
2^{|E|-k-3}-(|E|-k-3)
$$

ways.

Thus, the edge $e$ is in at most

$$
2^{|E|-k-3}+2^{|E|-k-3}-(|E|-k-3)=2^{|E|-k-2}-|E|+k+3
$$

interesting garlands.

Lemma 15. Assume that each part of $K(v)$ contains at least three vertices and $\langle E\rangle$ contains two triangles with a common edge e. If the edge $e$ is in $k$ different $\Xi_{2}$-subgraphs, then the edge $e$ is in at most $2^{|E|-k-3}-3|E|+3 k+15$ interesting garlands.

$\mathrm{P}$ r o o f. Let $\Delta_{1}$ and $\Delta_{2}$ be the edge sets of triangles from $\langle E\rangle$ that contain the edge $e$. Let edges $h_{1}, h_{2}, \ldots, h_{k}$ be such that the pair of edges $\left\{e, h_{i}\right\}$ induces a $\Xi_{2}$-subgraph for all $i=1,2, \ldots, k$. Define $E^{\prime}=E \backslash\left(\Delta_{1} \cup \Delta_{2} \cup\left\{h_{1}, h_{2}, \ldots, h_{k}\right\}\right)$ and $E_{\Delta}=\Delta_{1}=\Delta_{2}$.

Let $G$ be an arbitrary garland such that $e \in E(G)$. Define $F=E(G)$ and note that $h_{i} \notin F$ for all $i=1,2, \ldots, k$. Define $F^{\prime}=F \cap E^{\prime}$ and $F_{\Delta}=F \cap E_{\Delta}$. Note also that either $F_{\Delta}=\{e\}$, or $F_{\Delta}=\Delta_{1}$, or $F_{\Delta}=\Delta_{2}$, or $F_{\Delta}=\Delta$.

Consider an arbitrary $F^{\prime} \subseteq E^{\prime}$ and count the number of interesting garlands $G$ such that $e \in E(G)$ and $F^{\prime} \subseteq E(G)$. To do this, we estimate the number of ways to choose a subset $F_{\Delta} \subseteq \Delta$ such that $F^{\prime} \cup F_{\Delta}$ induces an interesting garland. If $\left|F^{\prime}\right| \neq 1$, then $F_{\Delta}$ can be chosen if four ways; if $\left|F^{\prime}\right|=1$, then $F \Delta \neq \Delta_{1}$ and $F \Delta \neq \Delta_{2}$. Let $f \in F^{\prime}$. Note that if the set $\{f, e\}$ induces an interesting garland $G^{\prime}$, then $G^{\prime}$ is of type $K(2,1)$ and $\{f\} \cup \Delta$ does not induce an interesting garland. Therefore, if $\left|F^{\prime}\right|=1$, then $F_{\Delta}$ can be chosen in at most one way.

Thus, the edge $e$ is in at most

$$
4 \cdot\left(2^{|E|-k-5}-(|E|-k-5)\right)+(|E|-k-5)=2^{|E|-k-3}-3|E|+3 k+15
$$

interesting garlands.

\section{Upper bound for the invariant $p t$ when $|E|$ is small}

The main goal of this section is to prove some upper bounds for the number of interesting garlands in the case where $E$ contains relatively small number of elements. 
Lemma 16. Assume that each active part of $K(v)$ contains at least three vertices, $\langle E\rangle$ contains a triangle, does not contain a coordinated subgraph of type $K(4,1)$, and $|E|=8$. Denote the edge set of the triangle by $\Delta$. If $\langle E \backslash \Delta\rangle$ contains at least two $\Xi_{2}$-subgraphs, then the number of interesting garlands does not exceed 46.

P r o o f. Let $X$ be the number of interesting garlands whose edge aggregates are subsets of $E^{\prime}$, and let $Y$ be the number of nonempty subsets of $E^{\prime}$ continuable outside $E^{\prime}$. Let $k$ be the number of edges $h \in E \backslash \Delta$ for which there exists an edge $h^{\prime}$ such that $h$ and $h^{\prime}$ induce a coordinated subgraph of type $K(2,1)$.

Case 1. Assume that there exist three edges $e, f_{1}, f_{2} \in E \backslash \Delta$ such that the pairs of edges $\left\{e, f_{1}\right\}$ and $\left\{e, f_{2}\right\}$ induce $\Xi_{2}$-subgraphs.

In this case, any continuable set can contain neither the set $\left\{e, f_{1}\right\}$ nor $\left\{e, f_{2}\right\}$. Consequently,

$$
Y \leqslant 2^{5}-1-2 \cdot 2^{3}+2^{2}=32-1-16+4=1 .
$$

By Lemma 3 , there are at most $2^{3}+1=9$ interesting garlands whose edge aggregates are subsets of $E^{\prime} \backslash\{e\}$.

Case 1.1. Assume that $E^{\prime} \backslash\left\{f_{1}, f_{2}\right\}$ induces a coordinated subgraph of type $K(3,1)$. Denote this subgraph by $H$. In this case, the edge $e$ is in exactly four interesting garlands whose edge aggregates are subsets of $E^{\prime}$. Consequently, $X \leqslant 9+4=13$.

If there exists an edge from the triangle adjacent to all edges from $H$, then either $\langle E\rangle$ contains a coordinated subgraph of type $K(4,1)$, which contradicts the lemma hypothesis, or any edge from $H$ does not induce a coordinated subgraph of type $K(2,1)$ with any edge from the triangle. Thus, $k \leqslant 2$. By Lemma 7 , the number of interesting garlands does not exceed

$$
2 \cdot 13+2 \cdot 19-5 \cdot 8+19+2 \leqslant 26+38-40+19+2=45 .
$$

Case 1.2. Define $E^{\prime \prime}=E^{\prime} \backslash\left\{f_{1}, f_{2}\right\}$ and assume that $E^{\prime \prime}$ does not induce a coordinated subgraph of type $K(3,1)$. In this case, there exist at most 5 interesting garlands whose edge aggregates are subsets of $E^{\prime \prime}$, and the edge $e$ is not in two (one-edge) of them. Consequently, the edge $e$ is in at most three interesting garlands whose edge aggregates are subsets of $E^{\prime}$. Therefore, $X \leqslant 9+3=12, k \leqslant 5$, and, using Lemma 7 , one can deduce that the number of interesting garlands does not exceed

$$
2 \cdot 12+2 \cdot 19-5 \cdot 8+19+5=24+38-40+24=46 .
$$

Case 2. Now consider the case where $\langle E\rangle$ contains two $\Xi_{2}$-subgraph without common edges. Let the pairs of edges $\left\{e_{1}, e_{2}\right\}$ and $\left\{g_{1}, g_{2}\right\}$ induce $\Xi_{2}$-subgraphs.

Estimate the number of nonempty continuable sets. Let $F \subseteq E^{\prime}$ be a nonempty continuable set, $F_{e}=F \cap\left\{e_{1}, e_{2}\right\}, F_{g}=F \cap\left\{g_{1}, g_{2}\right\}$, and let $F^{\prime}=F \backslash\left\{e_{1}, e_{2}, g_{1}, g_{2}\right\}$. Note that $F=F_{e} \cup F_{g} \cup F^{\prime}$. To obtain an upper bound, it suffices to count the number of ways to choose the sets $F_{e}, F_{g}$, and $F^{\prime}$. Note that $F^{\prime} \subseteq E^{\prime} \backslash\left\{e_{1}, e_{2}, g_{1}, g_{2}\right\}$ and then it can be chosen in

$$
2^{\left|E^{\prime} \backslash\left\{e_{1}, e_{2}, g_{1}, g_{2}\right\}\right|}=2^{5-4}=2
$$

ways. The set $F_{e}$ can be either empty or one-element because, by Lemma 9 , the edges $e_{1}$ and $e_{2}$ cannot be in the edge aggregate of any garland; so $F_{e}$ can be chosen in three ways. By analogy, the set $F_{g}$ can also be chosen in three ways. Excluding the empty set, one can conclude that $Y \leqslant 2 \cdot 3 \cdot 3-1=17$. 
Note that the set $E^{\prime} \backslash\left\{e_{1}\right\}$ contains a $\Xi_{2}$-subgraph and by Lemma 10, there are at most

$$
2^{\left|E^{\prime} \backslash\left\{e_{1}\right\}\right|-1}=2^{4-1}=8
$$

interesting garlands whose edge aggregates are subsets of $E^{\prime} \backslash\left\{e_{1}\right\}$. Note that three one-edge of them do not contain the edge $e_{2}$; consequently, $e_{2}$ is in at most 5 interesting garlands whose edge aggregates are subsets of $E^{\prime}$. By analogy, there are at most 8 interesting garlands whose edge aggregates are subsets of $E^{\prime} \backslash\left\{e_{2}\right\}$. Therefore, $X \leqslant 8+5=13$.

Thus, by Lemma 7 , the number of interesting garlands does not exceed

$$
2 \cdot 13+2 \cdot 17-5 \cdot 8+19+5=26+34-40+19+5=44
$$

and the lemma is proved.

Lemma 17. Assume that each part of $K(v)$ contains at least three vertices and $\langle E\rangle$ does not contain a coordinated subgraph of type $K(5,1)$. If $|E|=7$ and $\langle E\rangle$ contains at least three $\Xi_{2}$-subgraphs, then the number of interesting garlands does not exceed 41.

P r o o f. Assume that there exists an edge $e \in E$ that is in three $\Xi_{2}$-subgraphs. By Lemma 3, there exist at most $2^{5}+1=33$ interesting garlands whose edge aggregates are subsets of $E \backslash\{e\}$. By Lemma 12, one can deduce that $e$ is in at most $2^{7-4}=8$ interesting garlands. Therefore, there exist at most $33+8=41$ interesting garlands.

Now assume that there exists an edge $e \in E$ that is in exactly two $\Xi_{2}$-subgraphs. Since $\langle E\rangle$ does not contain a coordinated subgraph of type $K(5,1)$, by Lemma 12, one can deduce that $e$ is in at most $2^{7-3}-7+2+2=13$ interesting garlands. The graph $\langle E \backslash\{e\}\rangle$ contains a $\Xi_{2}$-subgraph and does not contain a coordinated subgraph of type $K(5,1)$. Thus, by Lemma 13, there exist at most $2^{5}-6+1=28$ interesting garlands whose edge aggregates are subsets of $E \backslash\{e\}$. Therefore, there exist at most $13+28=41$ interesting garlands.

It remains to consider the case when every edge is in at most one $\Xi_{2}$-subgraph. Let pairs of edges $\left\{e_{1}, e_{2}\right\},\left\{f_{1}, f_{2}\right\}$, and $\left\{g_{1}, g_{2}\right\}$ induce three different $\Xi_{2}$-subgraphs. Denote the single left edge by $h$. By Lemma 13, there exist at most $2^{5}-3 \cdot 6+6=20$ interesting garlands whose edge aggregates are subsets of $E \backslash\left\{e_{1}\right\}$.

Estimate the number of interesting garlands that contain the edge $e_{1}$. Let $H$ be the edge aggregate of such a garland. Define $H^{\prime}=H \cap\{h\}, H_{f}=H \cap\left\{f_{1}, f_{2}\right\}$, and $H_{g}=H \cap\left\{g_{1}, g_{2}\right\}$ and note that $H=\{e\} \cup H^{\prime} \cup H_{f} \cup H_{G}$. Note also that $H^{\prime}$ is empty or equal to $\{h\}$. The set $H_{f}$ can be chosen in three ways (because the edges $f_{1}$ and $f_{2}$ cannot be in the same garland). By analogy, the set $H_{g}$ can be chosen in three ways. Therefore, the set $H$ can be chosen in at most $2 \cdot 3 \cdot 3=18$ ways. Therefore, $e$ is in at most 18 interesting garlands, the total number of interesting garlands does not exceed $20+16=36$, and the lemma is proved.

Lemma 18. Assume that each part of $K(v)$ contains at least three vertices, $|E|=7$, and $\langle E\rangle$ does not contain a coordinated subgraph of type $K(4,1)$. If every edge is in at most three $\Xi_{2}$-subgraphs and $\langle E\rangle$ contains at least four $\Xi_{2}$-subgraphs, then the number of interesting garlands does not exceed 34 .

P r o o f. Assume that any edge is not in two $\Xi_{2}$-subgraphs. Then

$$
\xi_{2} \leqslant \frac{|E|}{2}=\frac{7}{2}<4,
$$

a contradiction. Consequently, there exists an edge that is in at least two $\Xi_{2}$-subgraphs. 
Case 1. Assume that there exist an edge $e$ that is in three $\Xi_{2}$-subgraphs. By Lemma 12, there exist at most $2^{6-3-1}+1-3=6$ interesting garlands such that $e$ is in them. Also, by Lemma 11, there are at most 28 interesting garlands whose edge aggregates are subsets of $E \backslash\{e\}$. Therefore, there exist at most $28+6=34$ interesting garlands.

Case 2. Assume that every edge is in at most two $\Xi_{2}$-subgraphs. Consider an edge $e$ that is in exactly two $\Xi_{2}$-subgraphs. Let edges $f_{1}$ and $f_{2}$ be such that the pairs of edges $\left\{e, f_{1}\right\}$ and $\left\{e, f_{2}\right\}$ induce $\Xi_{2}$-subgraphs.

Case 2.1. Assume that $E \backslash\left\{f_{1}, f_{2}\right\}$ contains a $\Xi_{2}$-subgraph. By Lemma 11, there exists at most $2^{4}-5+1=12$ interesting garlands whose edge aggregates are subsets of $E \backslash\left\{f_{1}, f_{2}\right\}$. Note that four one-edge garlands from them do not contain the edge $e$. Therefore, $e$ is at most in $12-4=8$ interesting garlands. The set $E \backslash\{e\}$ contains at least two $\Xi_{2}$-subgraphs and, by Lemma 13, there exist at most

$$
\max \left(2^{6-2}+2^{6-3}-6+5,2^{6-1}-3 \cdot 6+6\right)=\max \left(2^{4}+2^{3}-1,2^{5}-18+6\right)=\max (23,20)=23
$$

interesting garlands whose edge aggregates are subsets of $E \backslash\{e\}$.

Case 2.2. Assume that $E \backslash\left\{f_{1}, f_{2}\right\}$ does not contain a $\Xi_{2}$-subgraph. By Lemma 12 , the edge $e$ is in at most 13 interesting garlands.

Case 2.2.1. Assume that $E \backslash\{e\}$ contains two $\Xi_{2}$-subgraphs without common edges. In this case, by Lemma 13 , there exist at most $2^{6-1}-3 \cdot 6+6=20$ interesting garlands whose edge aggregates are subsets of $E \backslash\{e\}$. Therefore, there exists at most $20+13=33$ interesting garlands.

Case 2.2.2. It remains to consider the case when there exists an edge $h \in E \backslash\{e\}$ that is in two $\Xi_{2}$-subgraphs. Since every $\Xi_{2}$-subgraph contains $f_{1}$ or $f_{2}$ (otherwise, $E \backslash\left\{f_{1}, f_{2}\right\}$ contains a $\Xi_{2}$-subgraph), the pairs of edges $\left\{e, f_{1}\right\},\left\{e, f_{2}\right\},\left\{h, f_{1}\right\}$, and $\left\{h, f_{2}\right\}$ induce $\Xi_{2}$-subgraphs. This means that the edge aggregate of any garland is a subset of $E \backslash\left\{f_{1}, f_{2}\right\}$ or $E \backslash\{e, h\}$. Applying Lemma 3 to each of them, one can conclude that there exist at most 17 interesting garlands whose edge aggregates are subsets of each of them. Since three one-edge garlands were counted twice, the total number of interesting garlands does not exceed $17+17-3=31$, and the lemma is proved.

\section{Proof of Theorem 1}

The main goal of this section is to prove that a graph $K\left(n_{1}, n_{2}, n_{3}\right)$ is chromatically unique if

$$
n_{1} \geqslant n_{2} \geqslant n_{3} \geqslant 2, \quad n_{1}-n_{3}=5 \quad \text { and } \quad n_{1}+n_{2}+n_{3} \equiv 2 \quad(\bmod 3) .
$$

The bottom levels of the lattice $N P L(n, 3)$ in the case $n \equiv 2(\bmod 3)$ are shown in Fig. 5. The label above the covering relation shows how the number of edges changes, and the label under the covering relation shows how the invariant $p t$ changes. To prove the theorem, it suffices to verify that the graph $K(q+3, q+1, q-2)$ is chromatically unique for $q \geqslant 4$ and the graph $K(q+4, q-1, q-1)$ is chromatically unique for $q \geqslant 3$.

Proposition 1. The graph $K(q+3, q+1, q-2)$ is chromatically unique for $q \geqslant 4$.

P r o o f. By contradiction, assume that the graph $K(u)=K(q+3, q+1, q-2)$ is not chromatically unique. This means that there exists a graph $H$ such that the graphs $K(u)$ and $H$ are chromatically equivalent. Let $H$ be a graph obtained from the graph $K(v)$ by removing some 


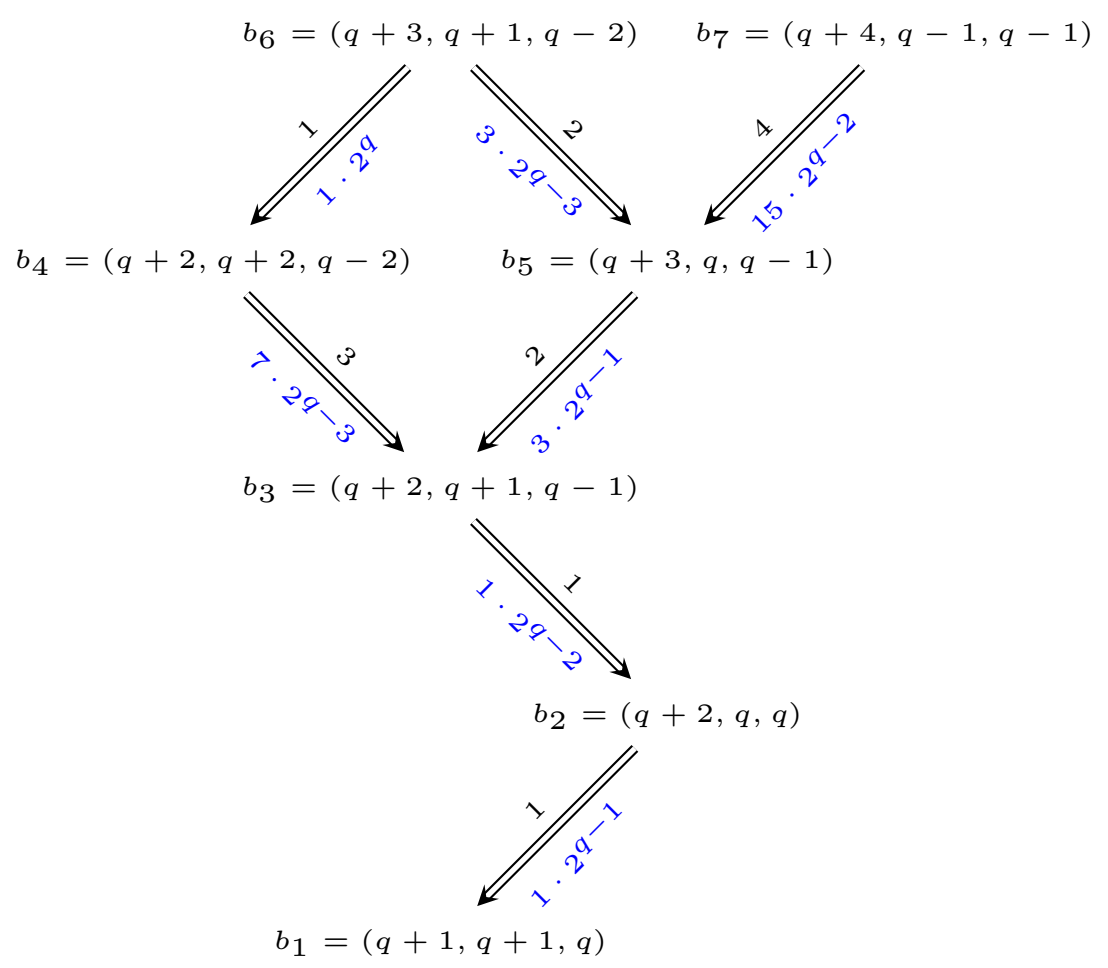

Figure 5. The bottom levels of the lattice $N P L(n, 3)$ in the case $n \equiv 2(\bmod 3)$

set of edges $E$. Consider the graph $K(v)$. Note that the cases $K(v)=K(q+2, q+2, q-2)$, $K(v)=K(q+3, q, q-1)$, and $K(v)=K(q+2, q+1, q-1)$ are impossible by Lemma 1.

Case 1. Assume that $K(v)=K(q+2, q, q)$ and note that, in this case, $|E|=5$. Calculate the difference of the invariant $p t$ :

$$
\Delta p t(H, K(v))=\Delta(K(u), K(v))=2^{q}+7 \cdot 2^{q-3}+2^{q-2}=17 \cdot 2^{q-3} .
$$

By Lemma 2, one can deduce that

$$
17 \cdot 2^{q-3} \leqslant 2^{5}-1=31,
$$

which implies $q \leqslant 3$, a contradiction.

Case 2. Assume that $K(v)=K(q+1, q+1, q)$. Note that, in this case, $|E|=6$. Calculate the difference of the invariant $p t$ :

$$
\Delta p t(H, K(v))=\Delta(K(u), K(v))=2^{q}+7 \cdot 2^{q-3}+2^{q-2}+2^{q-1}=21 \cdot 2^{q-3} .
$$

By Lemma 2, one can deduce that

$$
21 \cdot 2^{q-3} \leqslant 2^{6}-1=63,
$$

which implies $q \leqslant 4$; so it suffices to check the case $q=4$. In this case, $K(v)=K(5,5,4)$ and $\Delta p t(H, K(v))=42$. Note that, in this case, $E$ does not induce a coordinated subgraph of type $K(5,1)$ because such a subgraph is a one-element garland that destroys one part, a contradiction. Thus, by Lemma 3, one can obtain that there are at most $2^{5}+1=33$ interesting garlands, which contradicts $\Delta p t(H, K(v))=42$, and the proposition is proved.

To prove the chromatic uniqueness of the graph $K(q+4, q-1, q-1)$, we need the following two statements. 
Lemma 19. Let $E$ be a subset of edges of some complete tripartite graph $K(v)$. If any two triangles from $\langle E\rangle$ have a common edge, then there exists an edge that is in all triangles from $\langle E\rangle$.

Before proving this lemma, note that the condition on the graph $K(v)$ to be tripartite is necessary. Four triangles, any two of which have a common edge but no edge is common for all of them, are shown in Fig. 6.

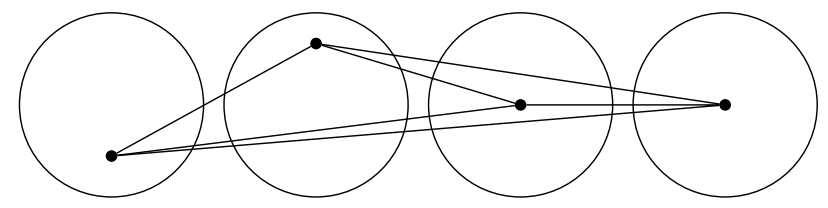

Figure 6. Four triangles, any two of which have a common edge but no edge is common for all of them.

$\mathrm{P} \mathrm{r}$ o o f. Let $\Delta_{1}$ and $\Delta_{2}$ be two triangles from $\langle E\rangle$ that have a common edge. Let $x, y_{1}$, and $z$ be vertices of the triangle $\Delta_{1}$ and let $x, y_{2}$, and $z$ be vertices of the triangle $\Delta_{2}$. Let us prove that if a triangle $\Delta$ from $\langle E\rangle$ have a common edge with the triangles $\Delta_{1}$ and $\Delta_{2}$, then $x z \in \Delta$.

By contradiction, assume that $x z \notin \Delta$. The triangles $\Delta_{1}$ and $\Delta$ have a common edge, without loss of generality, let it be the edge $y_{1} z$. Denote by $x^{\prime}$ the third vertex of $\Delta_{1}$ and note that the vertices $x$ and $x^{\prime}$ are in the same part of $K(v)$ because $K(v)$ is a tripartite graph. Note that, in this case, the edge set of $\Delta_{1}$ is $\left\{x^{\prime} y_{1}, x^{\prime} z_{1}, y_{1} z_{2}\right\}$ and it has an empty intersection with the edge set of $\Delta_{2}$ (see Fig. 7).

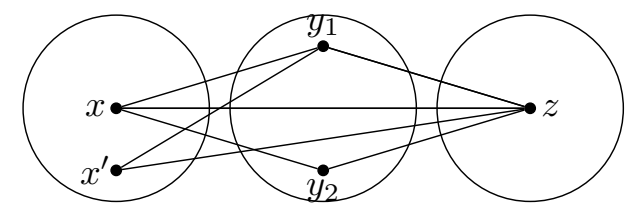

Figure 7. Three triangles in the tripartite graph that do not have a common edge

Proposition 2. If a graph $H$ is obtained by removing the edge set $E$ from the graph $K(4,4,3)$, then the graphs $H$ and $K(7,2,2)$ are not chromatically equivalent.

P r o o f. The graph $K(7,2,2)$ has $7 \cdot 2+7 \cdot 2+2 \cdot 2=32$ edges and the graph $K(4,4,3)$ has $4 \cdot 4+4 \cdot 3+4 \cdot 3=40$ edges; consequently, $|E|=40-32=8$.

Calculate the difference of the invariant $I_{3}$ :

$$
\begin{gathered}
I_{3}(K(7,2,2))=7 \cdot 2 \cdot 2=28, \\
I_{3}(K(4,4,3))=4 \cdot 4 \cdot 3=48, \\
20=\Delta I_{3}(K(4,4,3), K(7,2,2))=\Delta I_{3}(K(4,4,3), H)=\xi_{1}-\xi_{2}-2 \xi_{3}, \\
\xi_{1}=3 e_{12}+4 e_{13}+4 e_{23}=4|E|-e_{12}, \\
20=32-e_{12}-\xi_{2}-2 \xi_{3}, \\
\xi_{2}+2 \xi_{3}+e_{12}=12 .
\end{gathered}
$$

Note that every edge is in at most four $\Xi_{2}$-subgraphs. 
The inequality $e_{12} \leqslant 8$ implies $\xi_{2}+2 \xi_{3} \geqslant 4$. Note that $E \nsubseteq E_{i j}$ for all nonequal $i$ and $j$ from 1 to 3 because, otherwise, $\xi_{2}=\xi_{3}=0$, a contradiction. Assume that $e_{i j}=|E|-1$ for some $i$ and $j$. In this case, $\xi_{3}=0$ and $\xi_{2} \geqslant 5$. Denote the single edge from $E \backslash E_{i j}$ by $f$. Note that all $\Xi_{2}$-subgraphs must contain $f$; therefore, $\xi_{2} \leqslant 4$, a contradiction.

Calculate the difference of the invariant $p t$ :

$$
\begin{gathered}
p t(K(7,2,2))=2^{6}+2^{1}+2^{1}-3=65, \\
p t(K(4,4,3))=2^{3}+2^{3}+2^{2}-3=17 \\
\Delta p t(H, K(4,4,3))=\Delta p t(K(7,2,2), K(4,4,3))=65-17=48 .
\end{gathered}
$$

Note that $\langle E\rangle$ does not contain a coordinated subgraph of type $K(4,1)$ because, otherwise, such a subgraph forms a one-element garland that destroys one part, a contradiction.

Case 1. Assume that $\xi_{3}=0$.

Case 1.1. Assume that $e_{12}=6$. In this case, $\xi_{2}=6$.

Let edges $f$ and $g$ be not in $E_{12}$. Without loss of generality, assume that $f \in E_{23}$.

Note that any edge from $E$ except for $f$ and $g$ is not adjacent to any vertex from the part $V_{3}$. Since $\left|V_{3}\right|=3$, there exists a vertex in $V_{3}$ not incident to any edge from $E$. Consequently, any garland cannot destroy the part $V_{3}$.

By Lemma 3, there exist at most $2^{5}+1=33$ interesting garlands whose edge aggregates are subsets of $E_{12}$; so it suffices to prove that the number of interesting garlands $G$ such that $f \in E(G)$ or $g \in E(G)$ does not exceed 12 because, in this case, the total number of interesting garlands does not exceed $33+12=45<48$.

Case 1.1.1. Assume that edges $f$ and $g$ induce a $\Xi_{2}$-subgraph. Then there exist no garland $G$ such that $f \in E(G)$ and $g \in E(G)$. Note also that, since every edge is in at most four $\Xi_{2}$-subgraphs and every $\Xi_{2}$-subgraph contains $f$ or $g$, each of the edges $f$ and $g$ is in at least three $\Xi_{2}$-subgraphs.

Note that if $f$ is in a garland $G$ such that $|E(G)|>1$, then $|G|>1$; hence, $G$ must destroy some part. It cannot destroy the part $V_{3}$, so it must destroy $V_{1}$ or $V_{2}$; in both cases, $|E(G)| \geqslant 4$. Note that there exist at least two edges from $E_{12}$ such that $f$ induces a $\Xi_{2}$-subgraph with each of them. This means that there exist at most four edges $h \in E_{12}$ such that $f$ and $h$ can be in the edge aggregate of $G$. Note also that $E(G)$ must contain at least three edges from $E_{12}$, and these three edges can be chosen in $\left(\begin{array}{l}4 \\ 3\end{array}\right)=4$ ways. Therefore, $f$ is in at most five interesting garlands. By analogy, $g$ is in at most five interesting garlands, and the proof is complete in this case.

Case 1.1.2. Assume that edges $f$ and $g$ induce a coordinated subgraph of type $K(2,1)$. Note that, in this case, each of the edges $f$ and $g$ is in exactly three different $\Xi_{2}$-subgraphs. Note that if $f$ is in a garland $G$ that contains more than one element, then $G$ must destroy some part. Since it cannot destroy $V_{3}$, it must destroy $V_{1}$ or $V_{2}$; in both cases $|E(G)| \geqslant 4$.

Let $F \subseteq E_{12}$. Note that the following three statements are equivalent for the set $F$.

(1) The set $F \cup\{f\}$ induces an interesting garland.

(2) The set $F \cup\{g\}$ induces an interesting garland.

(3) The set $F \cup\{f, g\}$ induces an interesting garland.

The equivalence of these three statements follows from the following two observations. First, if $f$ is in some garland $G$, then $\{f\}$ forms an $E$-subgraph from $G$; and the same statement holds for $\{g\}$ and $\{g, f\}$. Therefore, the sets $F \cup\{f\}, F \cup\{g\}$, and $F \cup\{f, g\}$ induce a garland simultaneously. Second, denote by $V_{f}$ the vertex set of $\langle F \cup\{f\}\rangle$, by $V_{g}$ the vertex set of $\langle F \cup\{g\}\rangle$, and by $V_{g f}$ the vertex set of $\langle F \cup\{g, f\}\rangle$. Note that $V_{f} \cap\left(V_{1} \cup V_{2}\right)=V_{g} \cap\left(V_{1} \cup V_{2}\right)=V_{g f} \cap\left(V_{1} \cup V_{2}\right)$. This 
means that the sets $F \cup\{f\}, F \cup\{g\}$, and $F \cup\{f, g\}$ destroy the parts $V_{1}$ or $V_{2}$ simultaneously. Consequently, all of them induce interesting garlands or none of them does.

Note also that $F$ is chosen from a three-element set formed by edges $h \in E_{12}$ such that the edges $h$ and $f$ do not induce a $\Xi_{2}$-subgraph. Consequently, the set $F$ can be chosen in at most one way. Therefore, there exist at most $3 \cdot 1=3$ interesting garlands $G$ such that $f \in E(G)$ or $g \in E(G)$, and the prove is complete in this case.

Case 1.1.3. Assume that edges $f$ and $g$ do not induce a coordinated subgraph of type $K(2,1)$ and do not induce a $\Xi_{2}$-subgraph.

Note that, in this case, each of the edges $f$ and $g$ is in exactly three $\Xi_{2}$-subgraphs. Note that if $f$ is in a one-element garland $G$, then $G$ is a one-edge garland. Note also that if $f$ is in a garland $G$ that has more than one element, then $G$ must destroy $V_{1}$ or $V_{2}$, and then $|E(G)| \geqslant 4$. Since $f$ is in three $\Xi_{2}$-subgraphs, the set $E(G) \backslash\{f\}$ is chosen from a five-element set of edges. Consequently, $f$ is in at most

$$
\left(\begin{array}{l}
4 \\
3
\end{array}\right)+\left(\begin{array}{l}
4 \\
4
\end{array}\right)=4+1=5
$$

interesting garlands that contain more than one element. Therefore, $f$ is in at most six interesting garlands. By analogy, the edge $g$ is in at most six interesting garlands. Therefore, the number of interesting garlands whose edge aggregates contain $f$ or $g$ does not exceed 12 , and the lemma is proved in Case 1.1.

Case 1.2. Assume that $e_{12} \leqslant 5$. In this case, $\xi_{2} \leqslant 5$.

Case 1.2.1. Assume that there exists an edge $f \in E$ that is in four $\Xi_{2}$-subgraphs. Thus, by Lemma 12 , it is in at most $2^{8-4-1}-8+6=6$ interesting garlands. Note that the set $E \backslash\{f\}$ contains at least three $\Xi_{2}$-subgraphs and then, by Lemma 17 , there exist at most 41 interesting garlands whose edge aggregates are subsets of $E \backslash\{f\}$. Consequently, there are at most $6+41=47$ interesting garlands, a contradiction with $\Delta p t(H, K(v))=48$.

Case 1.2.2. Assume that there exists an edge $f \in E$ which is in exactly three $\Xi_{2}$-subgraphs. Thus, by Lemma 12 , it is in at most $2^{8-3-1}-8+5=13$ interesting garlands. Note that $E \backslash\{f\}$ contains at least four $\Xi_{2}$-subgraphs; so, by Lemma 18, there exist at most 34 interesting garlands whose edge aggregates are subsets of $E \backslash\{f\}$. Therefore, there exist at most $13+34=47$ interesting garlands, a contradiction with $\Delta p t(H, K(v))=48$.

Case 1.2.3. It remains to consider the case when every edge from $E$ is in at most two $\Xi_{2}$-subgraphs. Note that, since every $\Xi_{2}$-subgraph contains two edges, $\xi_{2} \leqslant 8$. If $e_{12}=5$, then every $\Xi_{2}$-subgraph must contain at least one of three edges from $E^{\prime}=E \backslash E_{12}$. Consequently, some edge from $E^{\prime}$ must be in three $\Xi_{2}$-subgraphs, a contradiction. Therefore, $e_{12} \leqslant 5$ and then $\xi_{2} \geqslant 8$. Thus, $\xi_{2}=8$ and $e_{12}$. Note that, in this case, every edge is in exactly two $\Xi_{2}$-subgraphs. Since $e_{12}=4$ and $\Xi_{2}$-subgraph cannot contain both edges from $E_{12}$, every $\Xi_{2}$-subgraph contain an edge from $E_{12}$.

Let $F$ be the edge aggregate of some garland. Note that $F$ does not contain edges of any $\Xi_{2}$-subgraph. Consequently, every $\Xi_{2}$-subgraph contains at least one edge from $E \backslash F$. Since every edge is in at most two $\Xi_{2}$-subgraphs, $8=\xi_{2} \leqslant 2|E \backslash F|$, which implies $|E \backslash F| \geqslant 4$, and then $|F| \leqslant 4$.

Assume that there exists a four-edge garland. Denote its edge aggregate by $F$ and define $F^{\prime}=E \backslash F$. Since $\left|F^{\prime}\right|=4$, there exists at most one four-edge garland whose edge aggregate is a subset of $F^{\prime}$. Note that every $\Xi_{2}$-subgraph contains one edge from $F$ and one edge from $F^{\prime}$. Estimate the number of four-edge garlands whose edge aggregates contain an edge from $F$ and an edge from $F^{\prime}$. Assume that such a garland exists. Denote its edge aggregate by $\tilde{F}$. Define $F=\left\{f_{1}, f_{2}, f_{3}, f_{4}\right\}$ and $F^{\prime}=\left\{f_{1}^{\prime}, f_{2}^{\prime}, f_{3}^{\prime}, f_{4}^{\prime}\right\}$. Let $f_{1} \in \tilde{F}$ and $f_{1}^{\prime} \in \tilde{F}$. Without loss of generality, assume that the pairs of edges $\left\{f_{1}, f_{2}^{\prime}\right\},\left\{f_{1}, f_{3}^{\prime}\right\},\left\{f_{1}^{\prime}, f_{2}\right\}$, and $\left\{f_{1}^{\prime}, f_{3}\right\}$ induce $\Xi_{2}$-subgraphs. Then 
$f_{4} \in \tilde{F}$. This means that every edge from $F$ is in at most one such four-edge garland and each such garland must contain exactly two edges from $F$; so the number of such garlands does not exceed $|F| / 2=2$. Therefore, the number of four-edge garlands does not exceed 4 .

Since $\langle E\rangle$ does not contain a triangle, an interesting three-edge garland must be of type $K(3,1)$ or $K(2,1) \dot{\cup} K(1,1)$. Since $e_{12}=4$, the inequality $e_{i j} \leqslant 4$ holds for all $i$ and $j$. Note that all three edges of a garland of type $K(3,1)$ must be in $E_{i j}$ for some $i$ and $j$. Since $\langle E\rangle$ does not contain a coordinated subgraph of type $K(4,1)$, any two garlands of type $K(3,1)$ cannot contain more than one common edge. Therefore, there exist at most two such garlands.

An interesting garland of type $K(2,1) \cup K(1,1)$ must destroy some part of $K(v)$. Since it can destroy only a part that contains three vertices, it must destroy $V_{3}$. Consequently, each vertex of $V_{3}$ must be incident to some edge from this garland. Therefore, its edge aggregate must be a three-element subset of $E \backslash E_{12}$. Since $\left|E \backslash E_{12}\right|=4$, such a subset can be chosen in $\left(\begin{array}{l}4 \\ 3\end{array}\right)=4$ ways; so the number of such garlands does not exceed 4.

Thus, there exist 8 one-edge garlands, at most

$$
\left(\begin{array}{l}
8 \\
2
\end{array}\right)=28
$$

interesting two-edge garlands, at most $4+2=6$ interesting garlands, and at most 4 four-edge interesting garlands. Therefore, there exist at most

$$
8+28+6+4=46<48
$$

interesting garlands. The proof is complete in Case 1.

Case 2. Assume that $\xi_{3}=1$.

Case 2.1. Assume that $e_{12}=6$. Then $\xi_{2}=4$ and $\left|E_{23}\right|=\left|E_{13}\right|=1$. Denote the single edge from $E_{23}$ by $e$ and the single edge from $E_{13}$ by $f$. Note that every $\Xi_{2}$-subgraph contains the edge $e$ or $f$. If $e$ is in three $\Xi_{2}$-subgraphs, then $\langle E\rangle$ contains a coordinated subgraph of type $K(4,1)$, a contradiction. By analogy, $f$ is in at most two $\Xi_{2}$-subgraphs. This means that 7 edges from $|E|$ form a configuration shown in Fig. 8.

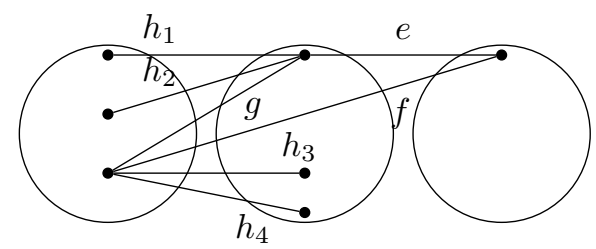

Figure 8. The case $e_{12}=6$ and $\xi_{3}=1$

Note that any garland cannot destroy the part $V_{3}$.

Assume that $e$ and $f$ are in some garland $G$ that contains more than one element. Note that $g \in E(G)$ and $h_{1}, h_{2}, h_{3}, h_{4} \notin E(G)$ because, otherwise, $\langle E\rangle$ contains at least two triangles. Consequently, $|E(G)| \leqslant 4$ and $\left|V(G) \cap V_{i}\right| \leqslant 2$ for all $i=1,2,3$. Therefore, $G$ cannot destroy any part, a contradiction. Thus, if $e$ and $f$ are in a garland $G$, then $|G|=1$. Note that there exists only one such garland, which is a triangle.

Note that $e$ is not in any coordinated subgraph of type $K(2,1)$. This means that there are only two one-element garlands whose edge aggregates contain $e$ : a triangle and a one-edge garland. Assume that $e$ is in some garland $G$ that contains more than one element and $f \notin E(G)$. Since $G$ must destroy some part and cannot destroy $V_{3}$, it destroys $V_{1}$ or $V_{2}$; hence, $|E(G)| \geqslant 4$. Note 
that $f, g, h_{1}, h_{2} \notin E(G)$, which implies $E(G)=E \backslash\left\{f, g, h_{1}, h_{2}\right\}$. Therefore, $e$ is in at most one interesting garland that contains more than one element and does not contain $f$. By analogy, $f$ is in at most one interesting garland that contains more than one element and does not contain $e$. Therefore, there exist at most 5 interesting garlands that contain $e$ or $f$.

By Lemma 3, there exist at most 33 interesting garlands whose edge aggregates are subsets of $E_{12}$. Therefore, there exist at most $33+5=38$ interesting garlands, which contradicts $\Delta p t(H, K(v))=48$.

Case 2.2. Assume that $e_{12} \leqslant 5$. In this case, $\xi_{2} \geqslant 5$. Note that any edge from the triangle cannot be in more than three $\Xi_{2}$-subgraphs.

Assume that some edge $e$ from the triangle is in three $\Xi_{2}$-subgraphs. By Lemma 14, one can deduce that $e$ is in at most $2^{8-2-3}-8+3+3=6$ interesting garlands. Since $\langle E \backslash\{e\}\rangle$ contains at least three $\Xi_{2}$-subgraphs, by Lemma 17 , there exist at most 41 interesting garlands whose edge aggregates are subsets of $E \backslash\{e\}$. Therefore, there exist at most $41+6=47$ interesting garlands, a contradiction.

Assume that some edge $e$ from the triangle is in two $\Xi_{2}$-subgraphs. By Lemma 14, one can deduce that $e$ is in at most $2^{8-2-2}-8+2+3=13$ interesting garlands. Since $\langle E \backslash\{e\}\rangle$ contains at least three $\Xi_{2}$-subgraphs, by Lemma 18 , there exist at most 34 interesting garlands whose edge aggregates are subsets of $E \backslash\{e\}$. Therefore, there exist at most $13+34=47$ interesting garlands, a contradiction.

By Lemma 16, there exists at most one $\Xi_{2}$-subgraph such that both its edges are not in the triangle. Taking into account that each edge of the triangle is in at most one $\Xi_{2}$-subgraph, one can deduce that $\xi_{2} \leqslant 3+1=4<5$, a contradiction.

Case 3. Consider the case $\xi_{3} \geqslant 2$.

If $\langle E\rangle$ contains two triangles without common edges, then, by Lemma 8, the number of interesting garlands does not exceed $2^{6}-12 \cdot 8+58=26<48$, a contradiction. Consequently, any two triangles from $\langle E\rangle$ have a common edge and, by Lemma 19, there exists an edge $e$ that is in all triangles. This implies $\xi_{3} \leqslant 3$. Define $E^{\prime}=E \backslash\{e\}$.

Note that there exist at most two $\Xi_{2}$-subgraph that do not contain the edge $e$. Indeed, any such $\Xi_{2}$-subgraph does not contain any edge from any triangle. Therefore, if there are at least three such subgraphs, then some two of them do not contain edges from the same triangle and, in this case, by Lemma 16, the number of interesting garlands does not exceed 46 .

Let $e$ be in $k$ different $\Xi_{2}$-subgraphs. Note that $k \leqslant 2$. Since $e$ is in $\xi_{3}$ triangles, there exist $\xi_{3}$ pairs of edges $\{h, f\}$ such that $h$ and $f$ induce a $\Xi_{2}$-subgraph in $\left\langle E^{\prime}\right\rangle$ (such pairs can be generated by removing the edge $e$ from a triangle from $\langle E\rangle)$. Therefore, $\left\langle E^{\prime}\right\rangle$ contains at least

$$
\xi_{2}+\xi_{3}-k=12-e_{12}-2 \xi_{3}+\xi_{3}-2=12-k-e_{12}-\xi_{3}=\xi_{2}^{\prime}
$$

different $\Xi_{2}$-subgraphs. Note that if $e_{12}=5$, then $\xi_{3} \leqslant 2$; if $e_{12} \leqslant 4$, then $\xi_{3} \leqslant 3$. In both cases, $e_{12}+\xi_{3} \leqslant 7$ and this implies $\xi_{2}^{\prime} \geqslant 12-k-7=5-k$.

Case 3.1. Assume that $k=2$. By Lemma 15 , the edge $e$ is in at most $2^{8-5}-3 \cdot 8+6+15=5$ interesting garlands. The graph $\left\langle E^{\prime}\right\rangle$ contains at least 3 different $\Xi_{2}$-subgraphs, therefore, by Lemma 17, there exist at most 41 interesting garlands whose edge aggregates are subsets of $E^{\prime}$. Thus, there exist at most $41+5=46<48$ interesting garlands, a contradiction.

Case 3.2. Assume that $k=1$. By Lemma 15, the edge $e$ is in at most $2^{8-4}-3 \cdot 8+3+15=10$ interesting garlands. The subgraph $\left\langle E^{\prime}\right\rangle$ contains at least four $\Xi_{2}$-subgraphs, therefore, by Lemma 18, there exist at most 34 interesting garlands whose edge aggregates are subsets of $E^{\prime}$. Thus, the number of interesting garlands does not exceed $10+34=44<48$, a contradiction. 
Case 3.3. It remains to consider the case when the edge $e$ is not in any $\Xi_{2}$-subgraph. Note that, in this case, $\xi_{2} \leqslant 2$.

If $e_{12}=5$, then $\xi_{3}=2$ and $\xi_{2}=12-2 \cdot 2-5=3>2$, a contradiction.

If $e_{12} \leqslant 3$, then $\xi_{3} \leqslant 3$ and $\xi_{2}=12-\left(e_{12}+2 \xi_{3}\right) \geqslant 12-9=3>2$, a contradiction.

Thus, it suffices to consider the case when $e_{12}=4$. In this case, $\xi_{3}=3$ and $\xi_{2}=2$. Consider the single edge that is not in any triangle. Note that this edge must be in two $\Xi_{2}$-subgraphs, which is impossible.

Now we are ready to prove the chromatic uniqueness of the graph $K(q+4, q-1, q-1)$.

Proposition 3. The graph $K(q+4, q-1, q-1)$ is chromatically unique for $q \geqslant 3$.

P r o o f. By contradiction, assume that the graph $K(u)=K(q+4, q-1, q-1)$ is not chromatically unique. This means that there exists a graph $H$ such that the graphs $K(u)$ and $H$ are chromatically equivalent. Let $H$ be a graph obtained from the graph $K(v)$ by removing some edge set $E$. Consider the graph $K(v)$. Note that the case $K(v)=K(q+3, q, q-1)$ is impossible by Lemma 1.

Case 1. Assume that $K(v)=K(q+3, q+1, q-2)$ and note that, in this case, $|E|=2$. Calculate the difference of the invariant $p t$ :

$$
\Delta p t(H, K(v))=\Delta p t(K(u), K(v))=15 \cdot 2^{q-2}-3 \cdot 2^{q-3}=27 \cdot 2^{q-3} .
$$

By Lemma 2, one can deduce that $27 \cdot 2^{q-3} \leqslant 2^{2}-1=3$, which implies $q<2$, a contradiction.

Case 2. Assume that $K(v)=K(q+2, q+2, q-2)$ and note that, in this case, $|E|=3$. Calculate the difference of the invariant $p t$ :

$$
\Delta p t(H, K(v))=\Delta p t(K(u), K(v))=15 \cdot 2^{q-2}-3 \cdot 2^{q-3}+2^{q}=35 \cdot 2^{q-3} .
$$

By Lemma 2, one can deduce that $33 \cdot 2^{q-3} \leqslant 2^{3}-1=7$, which implies $q<2$, a contradiction.

Case 3. Assume that $K(v)=K(q+2, q+1, q-1)$ and note that, in this case, $|E|=6$. Calculate the difference of the invariant $p t$ :

$$
\Delta p t(H, K(v))=\Delta p t(K(u), K(v))=15 \cdot 2^{q-2}+3 \cdot 2^{q-1}=21 \cdot 2^{q-2} .
$$

By Lemma 2, one can deduce that $21 \cdot 2^{q-2} \leqslant 2^{6}-1=63$, which implies $q \leqslant 3$, so it suffices to consider the case $q=3$. In this case, $K(v)=K(5,4,2)$ and $\Delta(H, K(v))=42$. Calculate the difference of the invariant $I_{3}$ :

$$
\begin{gathered}
\Delta I_{3}(K(v), K(u))=6(q-1), \\
\Delta I_{3}(K(v), H)=\xi_{1}-\xi_{2}-2 \xi_{3}, \\
\xi_{1}=(q-1) e_{12}+(q+1) e_{13}+(q+2) e_{23}=(q-1)|E|+2 e_{13}+3 e_{23}, \\
\xi_{2}+2 \xi_{3}=2 e_{13}+3 e_{23} .
\end{gathered}
$$

If $e_{13}=e_{23}=0$, then $E=E_{12}$ and each active part of $K(v)$ contains at least four vertices. Therefore, by Lemma 3 , there exist at most 33 interesting garlands, a contradiction. Thus, it suffices to consider the cases when $e_{13}>0$ or $e_{23}>0$. In both cases, $\xi_{2}+2 \xi_{3} \geqslant 2$.

Case 3.1. Assume that $\xi_{3}>0$ and edges $e_{1}, e_{2}, e_{3} \in E$ induce a triangle. Consider an arbitrary garland $G$ and define $F^{\prime}=E(G) \cap\left\{e_{1}, e_{2}, e_{3}\right\}$. Since $F^{\prime}$ can be one of five sets (empty, one-element or equal to $\left.\left\{e_{1}, e_{2}, e_{3}\right\}\right)$, there exist at most $5 \cdot 2^{6-3}-1=39<42$, a contradiction. 
Case 3.2. Now assume that $\xi_{3}=0$. This implies $\xi_{2} \geqslant 2$.

Case 3.2.1. Assume that there exists an edge $e$ that is in two $\Xi_{2}$-subgraphs. Let $f$ and $g$ be edges such that $\{e, g\}$ and $\{e, f\}$ induce $\Xi_{2}$-subgraphs. Let $G$ be a garland. Define $E^{\prime}=$ $E(G) \cap\{e, g, f\}$. Since $E^{\prime}$ can be one of five sets (empty, three one-element sets, and $\{g, f\}$ ), the number of garlands does not exceed $5 \cdot 2^{6-3}=40<42$, a contradiction.

Case 3.2.2. Now assume that there exist two $\Xi_{2}$-subgraphs without common edges. Let $e_{1}, e_{2}, f_{1}$, and $f_{2}$ be edges from $E$ such that $\left\{e_{1}, e_{2}\right\}$ and $\left\{f_{1}, f_{2}\right\}$ induce $\Xi_{2}$-subgraphs. Let $G$ be a garland. Define $E_{e}=E(G) \cap\left\{e_{1}, e_{2}\right\}, E_{f}=E(G) \cap\left\{f_{1}, f_{2}\right\}$, and $E^{\prime}=E(G) \backslash\left\{e_{1}, e_{2}, f_{1}, f_{2}\right\}$. Note that $E(G)=E_{e} \cup E_{f} \cup E^{\prime}$. Since $E_{e}$ can be chosen in three ways, $E_{f}$ can be chosen in three ways, and $E^{\prime}$ can be chosen in $2^{2}=4$ ways, the number of garlands does not exceed $3 \cdot 3 \cdot 4=36<42$, a contradiction.

Case 4. Assume that $K(v)=K(q+2, q, q)$ and note that, in this case, $|E|=7$. Calculate the difference of the invariant $p t$ :

$$
\Delta p t(H, K(v))=\Delta p t(K(u), K(v))=15 \cdot 2^{q-2}+3 \cdot 2^{q-1}+2^{q-2}=22 \cdot 2^{q-2} .
$$

By Lemma 2 , one can deduce that $22 \cdot 2^{q-2} \leqslant 2^{7}-1=127$, which implies $q \leqslant 4$. So it suffices to consider the cases when $q=4$ or $q=3$. If $q=4$, then $K(v)=K(6,4,4)$ and $\Delta p t(H, K(v))=88$. By Lemma 3, the number of interesting garlands does not exceed $2^{6}+1=65<88$, a contradiction. Thus, it suffices to consider the case $q=3$. In this case, $K(v)=K(5,3,3)$ and $\Delta p t(H, K(v))=44$. Note that $\langle E\rangle$ does not contain a coordinated subgraph of type $K(5,1)$.

Calculate the difference of the invariant $I_{3}$ :

$$
\begin{gathered}
\Delta I_{3}(K(v), K(u))=7 q-4, \\
\Delta I_{3}(K(v), H)=\xi_{1}-\xi_{2}-2 \xi_{3}, \\
\xi_{1}=q e_{12}+q e_{13}+(q+2) e_{23}=q|E|+2 e_{23}, \\
\xi_{2}+2 \xi_{3}=2 e_{23}+4 .
\end{gathered}
$$

If $\xi_{3}>0$, then, by Lemma 6 , the number of interesting garlands does not exceed

$$
2^{5}+2^{4}-3 \cdot 7+13=32+16-21+13=40<44,
$$

a contradiction. Therefore, $\xi_{3}=0$, which implies $\xi_{2}=2 e_{23}+4 \geqslant 4$. By Lemma 17, the number of interesting garlands does not exceed 41 , a contradiction.

Case 5. Assume that $K(v)=K(q+1, q+1, q)$ and note that, in this case, $|E|=8$. Calculate the difference of the invariant $p t$ :

$$
\Delta p t(H, K(v))=\Delta p t(K(u), K(v))=15 \cdot 2^{q-2}+3 \cdot 2^{q-1}+2^{q-2}+2^{q-1}=24 \cdot 2^{q-2} .
$$

Using Lemma 2, one can deduce that $24 \cdot 2^{q-1} \leqslant 2^{8}-1=255$, which implies $q \leqslant 6$; so it suffices to consider the cases when $q=5, q=4$, or $q=3$. The case $q=3$ is impossible by Proposition 2 .

If $q=5$, then $K(v)=K(6,6,5)$ and $\Delta p t(H, K(v))=192$. Since $\langle E\rangle$ dos not contain a coordinated subgraph of type $K(8,1)$, by Lemma 3 , the number of interesting garlands does not exceed $2^{7}+1=129<192$, a contradiction.

It remains to prove the proposition in the case $q=4$. In this case, $K(v)=K(5,5,4)$ and $\Delta p t(H, K(v))=96$. 
Calculate the difference of the invariant $I_{3}$ :

$$
\begin{gathered}
\Delta I_{3}(K(v), K(u))=8 q-4, \\
\Delta I_{3}(K(v), H)=\xi_{1}-\xi_{2}-2 \xi_{3} \\
\xi_{1}=q e_{12}+(q+1) e_{13}+(q+1) e_{23}=(q+1)|E|-e_{12} \\
8 q-4=8(q+1)-e_{12}-\xi_{2}-2 \xi_{3}, \\
12=\xi_{2}+2 \xi_{3}+e_{12} .
\end{gathered}
$$

If $\xi_{3}>0$, then, by Lemma 6 , the number of interesting garlands does not exceed

$$
2^{6}+2^{5}-3 \cdot 8+13=85<96,
$$

a contradiction. Thus, $\xi_{3}=0$, which implies $\xi_{2}+e_{12}=12$. Note that $e_{12} \leqslant 8$. If $e_{12}=8$, then $E=E_{12}$, which implies $\xi_{2}=0$, a contradiction. Thus, $e_{12} \leqslant 7$ and $\xi_{2} \geqslant 5$.

If there exist an edge $e$ that is in at least two $\Xi_{2}$-subgraphs, then, since $\langle E\rangle$ does not contain a coordinated subgraph of $K(6,1)$, by Lemma 12, the edge $e$ is in at most $2^{5}-8+2+2=28$ interesting garlands. By Lemma 3, the number of interesting garlands whose edge are subsets of $E \backslash\{e\}$ does not exceed $2^{6}+1=65$, so the total number of interesting garlands does not exceed $65+28=93<96$, a contradiction. Therefore, every edge is in at most one $\Xi_{2}$-subgraphs, which implies $\xi_{2} \leqslant 4$, a contradiction.

The proof of the theorem follows from Propositions 1 and 3.

\section{Conclusion}

In this paper, the chromatic uniqueness of a complete tripartite graph $K\left(n_{1}, n_{2}, n_{3}\right)$ is proved for $n_{1} \geqslant n_{2} \geqslant 3 \geqslant 2$ and $n_{1}-n_{3} \leqslant 5$. Also, some properties of the number of partitions of the vertex set of a graph $G$ into $t$ independent sets are established. Both problems, the chromatic uniqueness, and properties of the invariant $p t$, are still challenging open problems.

\section{Acknowledgements}

The author is grateful to his scientific advisor prof. V.A. Baransky for constant attention and remarks.

\section{REFERENCES}

1. Asanov M. O., Baransky V.A., Rasin V.V. Diskretnaya matematika: grafy, matroidy, algoritmy [Discrete Mathematics: Graphs, Matroids, Algorithms]. Saint-Petersburg: "Lan", 2010. 364 p. (in Russian)

2. Baransky V. A., Koroleva T. A. Chromatic uniqueness of certain complete tripartite graphs. Izv. Ural. Gos. Univ. Mat. Mekh. Inform., 2010. Vol. 74, Suppl. 12. P. 5-26. (in Russian)

3. Baransky V. A., Koroleva T. A., Senchonok T. A. On the partition lattice of all integers. Sib. Èlektron. Mat. Izv., 2016. Vol. 13. P. 744-753. DOI: 10.17377/semi.2016.13.060 (in Russian)

4. Baranskii V. A., Sen'chonok T. A. Chromatic uniqueness of elements of height $\leq 3$ in lattices of complete multipartite graphs. Proc. Steklov Inst. Math., 2012. Vol. 279. P. 1-16. DOI: 10.1134/S0081543812090015

5. Brylawski T. The lattice of integer partitions. Discrete Math., 1973. Vol. 6, No. 3. P. 210-219. DOI: 10.1016/0012-365X(73)90094-0

6. Dong F. M., Koh K.M., Teo K.L. Chromatic Polynomials and Chromaticity of Graphs. Hackensack: World Scientific, 2005. 384 p. DOI: 10.1142/5814 
7. Farrell E.J. On chromatic coefficients. Discrete Math., 1980. Vol. 29, No. 3. P. $257-264$. DOI: $10.1016 / 0012-365 \mathrm{X}(80) 90154-5$

8. Gein P. A. About chromatic uniqueness of complete tripartite graph $K(s, s-1, s-k)$, where $k \geq 1$ and $s-k \geq 2$. Sib. Elektron. Mat. Izv., 2016. Vol. 13. P. 331-337. DOI: 10.17377/semi.2016.13.027 (in Russian)

9. Gein P. A. About chromatic uniqueness of some complete tripartite graphs. Sib. Èlektron. Mat. Izv., 2017. Vol. 14. P. 1492-1504. DOI: 10.17377/semi.2017.14.129 (in Russian)

10. Gein P. A. On garlands in $\chi$-uniquely colorable graphs. Sib. Èlektron. Mat. Izv., 2019. Vol. 16. P. 17031715. DOI: $10.33048 /$ semi.2019.16.120

11. Koh K. M., Teo K. L. The search for chromatically unique graphs. Graphs Combin., 1990. Vol. 6, Suppl. 3. P. 259-285. DOI: 10.1007/BF01787578

12. Koroleva T. A. Chromatic uniqueness of some complete tripartite graphs. I. Trudy Inst. Mat. $i$ Mekh. UrO RAN, 2007. Vol. 13, Suppl. 3. P. 65-83. (in Russian)

13. Koroleva T. A. Chromatic uniqueness of some complete tripartite graphs. II. Izv. Ural. Gos. Univ. Mat. Mekh. Inform., 2010. Vol. 74. P. 39-56. (in Russian)

14. Li N.Z., Liu R. Y. The chromaticity of the complete $t$-partite graph $K\left(1, P_{2} \ldots p_{t}\right)$. J. Xinjiang Univ. Natur. Sci., 1990. Vol. 7, No. 3. P. 95-96.

15. Senchonok T. A. Chromatic uniqueness of elements of height 2 in lattices of complete multipartite graphs. Trudy Inst. Mat. i Mekh. UrO RAN, 2011. Vol. 17, No. 3. P. 271-281. (in Russian)

16. Zhao H. Chromaticity and Adjoint Polynomials of Graphs. Zutphen, Netherlands: Wöhrmann Print Service, 2005. 179 p.

17. Zhao H., Li X., Zhang Sh., Liu R. On the minimum real roots of the $\sigma$-polynomials and chromatic uniqueness of graphs. Discrete Math., 2004. Vol. 281, No. 1-3. P. 277-294. DOI: 10.1016/j.disc.2003.06.010 